\title{
A Check List of \\ Plant Rusts in Canada
}

by

A. M. Brown

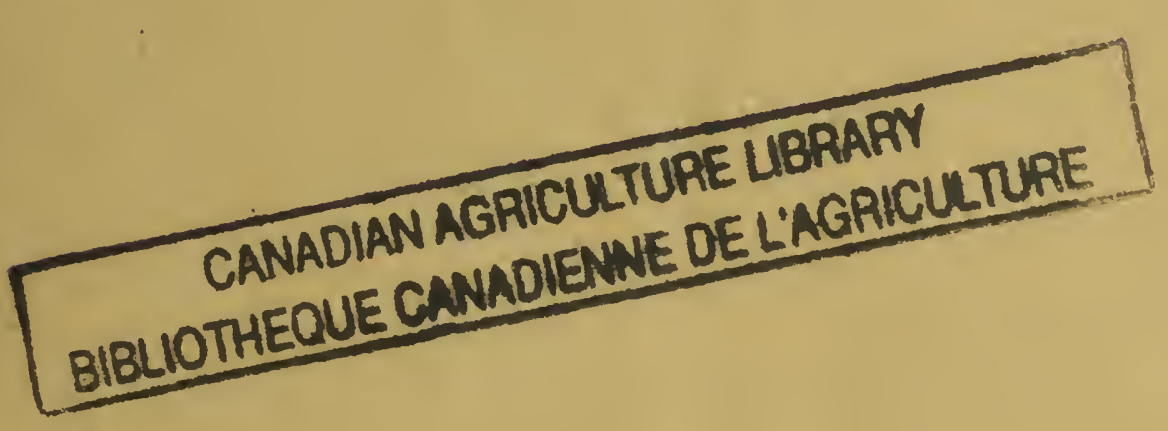

Canada Department of Agriculture Ottawa, Ontario 


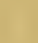
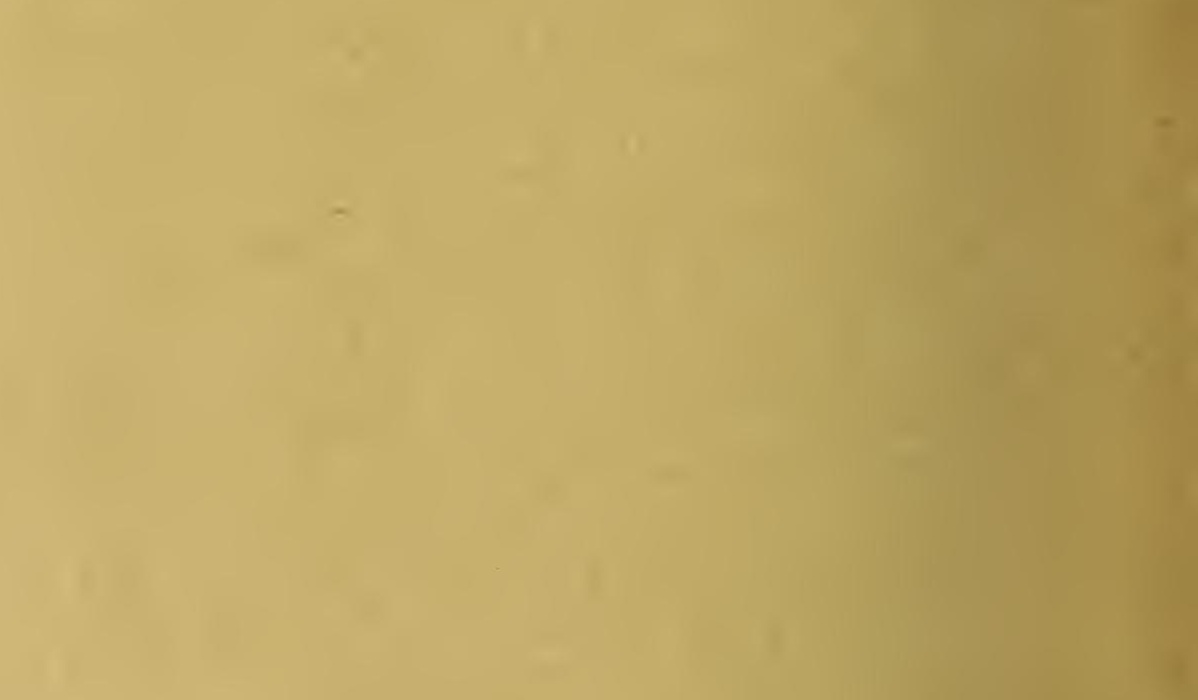


\section{A Check List of \\ Plant Rusts in Canada}

by

A. M. Brown

Plant Pathology Laboratory

Winnipeg, Manitoba

Botany and Plant Pathology Division

Canada Department of Agriculture

Ottawa, Ontario 



\section{FOREWORD}

This check list of the Plant Rusts in Canada has been prepared from records published up to 1952 .

Many of the rusts have been recorded for the same area in more than one publication and for this reason some of the records have been omitted.

The rusts are arranged in alphabetical order and the epithets have been taken mostly from the Manual of the Rusts in United States and Canada by J. C. Arthur. The names of the hosts are followed by the names of the provinces or other regional areas in which the rusts were collected and these, in turn, are followed by reference numbers. These numbers refer to the publications from which the records were taken.

A list of hosts and the rusts that may be expected to occur on them has also been included. In this list the hosts are listed more completely than in the section devoted to the rusts. The names of the hosts were taken for the most part from Gray's Manual of Botany, 8th ed., but in some cases it was found advisable to follow Rydberg's Flora of the Prairies and Plains of Central North America. The names of the grass hosts were taken mostly from Hitchcock's Manual of the Grasses of the United States.

A. M. BROWN

Plant Pathology Laboratory Winnipeg, Manitoba 
Digitized by the Internet Archive in 2012 with funding from

Agriculture and Agri-Food Canada - Agriculture et Agroalimentaire Canada 


\section{SUPPLEMENTARY NOTE}

Mr. Brown's "A Check List of Plant Rusts in Canada" is compiled for the most part from published records, but he has included some of his own observations as well. In some instances records of certain rusts and significant host and range extensions of other species given in the annual reports of the "Canadian Plant Disease Survey" or other publications have been overlooked. Also, since Mr. Brown compiled his list several comprehensive studies on the taxonomy of rusts occurring in Canda have appeared. To increase the usefulness of the list, the text covering certain species was revised and the pertinent publications have been added as an addendum to the references. Besides, in the interests of greater clarity, notes about a few rusts have been added in parentheses.

\section{L. Conners}

Senior Mycologist 



\section{THE PLANT RUSTS AND THEIR HOSTS}

For each rust are shown the known stages, the hosts on which they have been collected, the province, territory or district in which the collections have been made and one or more references to the literature.

Aecidium columbiense Ell. \& Ev.

O-I On Hieracium albiflorum Hook.; B.C. (1).

Aecidium graebnerianum P. Henn.

(See also Puccinia praegracilis)

I On Habenaria dilatata (Pursh) Hook; Que. (29).

H. gracilis Wats.; B.C. (1).

H. psychodes (L.) Sw.; Que. (29).

Habenaria sp.; N. S. (29).

Aecidium ligustici Ell. \& Ev.

O-I On Ligusticum scothicum L.; Que. (1).

Aecidium mitellae Ell. \& Ev.

I On Mitella nuda L.; Nfld. (1).

Chrysomyxa arctostaphyli Diet.

III On Arctostaphylos urva-ursi (L.) Spreng.; B.C., Alta., Yukon, Sask. (1), Man. (2), Mack., Ont., Que. (27).

Chrysomyxa cassandrae (Peck \& Clint.) Tranz.

(See C. ledi var. cassandrae)

Chrysomyxa chiogenis Diet.

O-I On Picea glauca (Moench) Voss; Ont. (culture) (32).

P. mariana (Mill.) B.S.P.; Ont. (culture) $(32,35)$.

II-III On Chiogenes hispidula (L.) T. \& G.; B.C. (35), Ont., Que., N.S。 (27), Nfld. (1, 35).

Chrysomyxa empetri (Pers.) Schroet.

O-I On Picea engelmanni (Parry) Engelm.; B.C. (27).

P. glauca (Moench) Voss; Que. (33), N.S. (27), Nfld. (35).

P. rubens Sarg.; Que. (culture) (33).

II-III On Empetrum nigrum L.; B.C., Que. (1), Alta., N.S., Nfld. (33),

Yukon, Mack., Frank., Man. (27), Keew., Labr. (35).

E. atropurpureum Fern. \& Wieg.; Que. (33).

E. eamesii Fern. \& Wieg.; Nfld. (33).

Chrysomyxa ledi (Alb. \& Schw.) de Bary

O-I On Picea mariana (Mill.) B.S.P.; Sask., Ont., N.S. (1)

$P$. pungens Engelm.; Ont. (1)

P. rubens Sarg.; Ont., N.S. (1)

C. ledi var. cassandrae (Peck \& Clint.) Savile

O-I On Picea glauca (Moench) Voss; N.S. (27).

P. rubens Sarg.; N.S. (27).

II-III On Chamaedaphne calyculata (L.) Moench; Man., Ont., N.S. (1),

Yukon, Mack., Que. (27).

C. ledi var. glandulosi Savile

O-I On Picea engelmanni (Parry) Engelm.; B.C. (35)

II-III On Ledum glandulosum Nutt.; B.C. (35) 
C. ledi var. groenlandici Savile

O-I On Picea mariana (Mill.) B.S.P.; N.S. (27).

P. rubens Sarg.; N.S. (27).

II-III On Ledum groenlandicum Oeder; B.C., Sask., Ont., N.S. (1), Mack., Que. (27), Alta., Nfld. (35).

C. ledi var. ledi

II-III On Ledum palustre L. var. decumbens Ait.; Mack. (35).

C. ledi var. rhododendri (de Bary) Savile

II-III On Rhododendron lapponicum L.; Mack. (27), B.C., Man., Nfld. (35).

C. ledi var. vaccinii Ziller

II-III On Vaccinium parvifolium Smith; B.C. (35).

Chrysomyxa ledicola (Peck) Lagerh.

O-I On Picea engelmanni (Parry) Engelm.; Alta (1)

P. glauca (Moench) Voss (P. canadensis (Mill.) B.S.P.); Alta., Sask., Que., N.B., N.S. (1), Man. (2), Mack., Ont. (27), B.C., Nfld. (35).

P. glauca var. albertiana (S. Brown) Sarg.; Yukon (27), B.C. (35).

P. mariana (Mill.) B.S.P.; Alta., Man., Que., N.S., Nfld. (1), Sask., Ont. (2).

P. pungens Engelm.; Ont. (1), Alta., Man., Que., P.E.I. (27).

P. rubens Sarg.; N.S., P.E.I. (1), Que. (27).

P. sitchensis (Bong.) Carr.; B.C. (35).

II-III On Ledum groenlandicum Oeder; B.C., Alta., Yukon, Sask., Man., Nfld. (1) Mack., Que. (27).

L. palustre L. var. decumbens Ait.; Labr. (1), Yukon, Mack., Frank., Keew. (27).

Chrysomyxa monesis Ziller

O-I On Picea sitchensis (Bong.) Carr.; B.C. (36).

II-III On Moneses uniflora (L.) Gray; B.C. $(35,36)$.

Chrysomyxa pirolata (Koern.) Wint. (C. pyrolae (DC.) Rostr. nom. nud.)

O-I On Picea glauca (Moench) Voss (P. canadensis (Mill.) B.S.P.); Man. (1), Ont., Que., N.S. (27), Mack. (35).

P. glauca var. albertiana (S. Brown) Sarg.; B.C. (27).

P. mariana (Mill.) B.S.P.; B.C., N.S. (27).

II-III On Moneses uniflora (L.) Gray.; Alta., Que. (1), Man. (27).

Pyrola americana Sweet; N.S. (1), Man. (2), B.C., Sask., Ont., Que. (27).

P. asarifolia Michx.; B.C., Sask. (1), Man. (27).

P. bracteata Hook.; B.C. (1).

$P$. chlorantha Sw.; (P. virens Schweigg.), Alta. (1), Mack., Que. (27), B.C. (35).

P. elliptica Nutt.; Alta. (1), Man., Que., N.S. (27).

$P$. grandiflora Radius; Yukon, Keew., Frank. (27).

P. minor L.; Que. (27).

P. picta Smith; B.C. (35).

P. secunda L., ; Que., Nfld. (1), B.C., Mack., Alta., Man. (27).

P. uliginosa. T. \& G.; N.S. (1). 
Chrysomyxa weirii Jacks.

III On Picea engelmanni (Parry) Engelm.; B.C. (1).

P. rubens Sarg.; N.B. (9).

P. sitchensis (Bong.) Carr.; B.C. (27).

Picea sp.; N.S. (27).

Chrysomyxa woronini Tranz.

O-I On Picea glauca (Moench) Voss; Que. (27), B.C., Nfid. (35).

P. mariana (Mill.) B.S.P.; Nfld. (35).

III On Ledum groenlandicum Oeder; Nfld. (35).

L. palustre var. decumbens Ait.; Yukon (27).

Coleosporium campanulae (Pers.) Lév.

II-III On Campanula rapunculoides L.; Que. (15), Ont. (14)

C. persicifolia L.; B.C. (7).

Campanula spp.; B.C. (7), N.S. (23), Ont. (13).

Coleosporium madiae Cooke

II-III On Madia sativa Molina; B.C. (1).

Coleosporium solidaginis (Schw.) Thuem.

O-I On Pinus banksiana Lamb.; Alta., Man., Sask. (25), Que. (6). P. contorta Dougl.; Alta. (1).

II-III Common on Aster spp. and Solidago spp. in most provinces.

Coleosporium viburni Arth.

II-III On Viburnum cassinoides L.; Ont. (1), N.S. (14), N.B. (13).

$V$. lentago L.; Man., (3), Ont. (14).

Cronartium coleosporioides (Diet. \& Holw.) Arth.

O-I On Pinus contorta Dougl.; Que., N.B. (21), B.C. (1). P. ponderosa Dougl.; B.C. (1).

Cronartium comandrae Peck

O-I On Pinus banksiana Lamb.; Alta. (1), Sask. (2), Ont. (5).

P. contorta Dougl.; Alta. (1).

P. ponderosa Dougl.; B.C. (1).

Pinus spp.; Man. (21).

II-III On Comandra livida Richards.; Man. (2), Que. (1).

C. pallida A. DC.; Alta., Sask. (25), B.C., N.W.T. (1), Man., Sask. (2).

C. umbellata (L.) Nutt.; Ont., Que. (1).

Cronartium comptoniae Arth.

O-I On Pinus banksiana Lamb.; Man., Ont. (2).

II-III On Myrica gale L.; B.C., N.S., Ont. (1).

Cronartium flaccidum (Alb. \& Schw.) Wint.

III On Impatiens balsamina L.; P.E.I. (1).

Cronartium ribicola J. C. Fisch.

O-I On Pinus spp.; B.C., Que., N.S., P.E.I. (12).

II-III On Grossularia spp.; Man. (15), N.S. (22), P.E.I. (20), B.C. (18).

Ribes spp.; B.C., Que., N.B., P.E.I. (12), Man., Ont. (15).

Cumminsiella sanguinea (Peck) Arth.

O-I-II-III On Mahonia sp.; B.C. (18), Que., Ont. (21).

Endophyllum sempervivi (Alb. \& Schw.) de Bary.

O-III On Sempervivum tectorum L.; Ont., B.C. (6).

$72559-2 \frac{1}{2}$ 
Frommea obtusa (Strauss) Arth.

O-I-II-III On Potentilla canadensis L.; N.S., Ont. (1).

Gymnoconia peckiana (Howe) Trott.

O-I-III On Rubus acaulis Michx.; Sask. (2).

R. articus L.; Sask. (25), Alta. (1).

R. nigrobaccus Bailey; Ont. (1).

R. randii (Bailey) Rydb.; N.S. (1).

R. triflorus Richards.; Man. (2).

Rubus spp.; B.C., Ont., Que., N.B., N.S. (23).

Gymnosporangium aurantiacum Chev. (legitimate name G. cornutum Arth.)

O-I On Sorbus americana Marsh.; Ont., N.B., Nfld. (1), Man. (2).

S. scopulina Greene; Man., Ont., Que. (1).

Gymnosporangium betheli Kern

O-I On Crataegus chrysocarpa Ashe.; Sask. (25), Man. (2).

C. douglasii Lindl.; B.C. (1).

Crataegus spp.; Morden, Man. Dr. W. L. Gordon.

III On Juniperus scopulorum Sarg.; Man. (16).

Gymnosporangium clavariaeforme (Jacq.) DC.

O-I On Amelanchier alnifolia Nutt.; Man. (1).

A. canadensis (L.) Medic.; N.S. (1), Ont. (6).

A. spicata (A. ?stolonifera Wieg.); N.S. (17).

Crataegus chrysocarpa Ashe.; Sask. (25).

Crataegus spp.; P.E.I., B.C. (12), Ont., Que. (23).

Cydonia oblonga Mill.; N.S. (23).

Pyrus communis L.; N.S. (23).

III On Juniperus communis L. var. depressa Pursh; Ont., Que. (5), N.S. (4).

J. communis L. var. hibernica Gord.; P.E.I. (10).

J. communis L.; N.S. (16).

Gymnosporangium clavipes Cooke \& Peck

O-I On Amelanchier spp.; B.C., Man., Sask., Que., N.B., N.S., Ont., common.

Aronia arbutifolia (L.) Ell.; N.B. (1).

Crataegus punctata Jacq.; Ont. (1).

Crataegus spp.; Man. (2), Ont., Que., N.B., N.S. (23), Sask. (6).

Cydonia oblonga Mill.; Ont., N.S. (23).

Malus pumila Mill.; Ont., Que., N.B., N.S. (23).

Cotoneaster sp.; Man. (20).

Cydonia sp.; Ont. (16), N.S. (20).

III On Juniperus communis L. var. depressa Pursh; Ont., Que. (5).

$J$. horizontalis Moench; Que. (5).

J. communis L.; N.S. (17), Que. (12).

Juniperus spp.; Ont. (6).

Gymnosporangium corniculans Kern

O-I. On Amelanchier alnifolia Nutt.; Man. (1), Sask. (25).

A. sanguinea (Pursh) DC.; Ont. (1).

III On Juniperus horizontalis Moench $f$. douglasii; Rehd. Man. (16). $J$. virginiana L.; Man. (16). 
Gymnosporangium globosum Farl.

O-I On Crataegus coccinea L.; Ont. (1).

C. punctata Jacq.; Ont. (1).

C. sanguinea (Pursh) DC.; Ont. (5).

C. tomentosa L.; Ont., Que. (1).

Crataegus sp.; Sask. (2).

Malus pumila Mill.; Ont., Que. (23).

III On Juniperus virginiana L.; Ont. (1), Que. (10).

$J$. horizontalis Moench; Sask. (2).

$J$. fragrans (cult.); Ont. (5)

Juniperus spp.; Ont. (6).

Gymnosporangium juniperinum (L.) Mart.

(legitimate name, G. tremelloides Hartig)

O-I On Sorbus occidental is (Wats.) Greene; Alta., B.C. (11).

S. scopulina Greene; B.C. (1).

S. sitchensis Roem.; B.C. (1).

III On Juniperus sibirica Burgsd.; Alta. (1).

Gymnosporangium juniperi-virginianae Schw.

O-I On Malus coronaria (L.) Mill.; Ont. (1).

M. pumila Mill.; Ont. (23).

M. sylvestris (L.) Mill.; Ont. (1).

III On Juniperus virginiana L.; Ont. (10).

Gymnosporangium juvenescens Kern

O-I On Amelanchier alnifolia Nutt.; Alta. (1), Sask., Man. (25).

A. florida Lindl.; B.C. (1).

A. pumila Nutt.; Alta. (1).

III On Juniperus horizontalis Moench; Sask. (25), B.C. (24).

Gymnosporangium nelsoni Arth.

O-I On Amelanchier alnifolia Nutt.; Alta., B.C. (1), Man. (2). A. florida Lindl.; B.C. (19).

III On Juniperus scopulorum Sarg.; Alta. (1), B.C. (19).

Gymnosporangium nidus-avis Thaxt.

O-I On Amelanchier intermedia Spach; Ont. (1).

III On Juniperus virginiana L.; Ont. (12).

Gymnosporangium nootkatense (Trel.) Arth.

O-I On Malus rivularis (Dougl.) Roem.; B.C. (1).

Sorbus occidentalis (Wats.) Greene; B.C. (1).

Gymnosporangium tubulatum Kern

O-I On Crataegus sp.; B.C. (15).

Hyalopsora aspidiotus (Peck) Magn.

O-I On Abies balsamea (L.) Mill.; Ont., Que. (1).

II-III On Phegopteris dryopteris (L.) Fée; B.C., Ont., Que. (1).

Hyalopsora polypodii (Pers.) Magn.

II-III On Filix fragilis (L.) Gilib.; B.C., Ont. (1), Sask. (25).

Kuehneola uredinis (Link) Arth.

O-I-II-III On Rubus procumbens Muhl; Ont. (1.)

R. pubescens Raf.; N.S. (1). 
Melampsora abietis-canadensis (Farl.) C. A. Ludwig

O-I On Tsuga canadensis (L.) Carr.; N.S., Que. (1).

II-III On Populus grandidentata Michx. N.S., Ont., Que. (1), P.E.I. (14).

P. tremuloides Michx.; Ont., Que. (1).

Melampsora abieti-capraearum Tubeuf

O-I On Abies balsamea (L.) Mill.; Sask. (2), N.S. (1).

II-III On Salix alba L. var. vitellina L.; Ont. (18).

S. bebbiana Sarg.; N.B. (18).

S. candida Flugge; Sask. (2).

S. cordata Muhl.; Man. (2).

S. purpurea L. var. gracilis Anderss.; Man. (14).

S. rubra Richards.; Ont. (14).

Melampsora albertensis

O-I On Pseudotsuga taxifolia (Poir.) Britt.

(P. mucronata (Raf.) Sudw.; B.C. (1), ?B.C., Alta. (37)

II-III On Populus tremuloides Michx.; B.C., Alta. (1, 37).

Melampsora arctica Rostr.

II-III On Salix anglorum Cham.; Alta., B.C. (1).

S. arbusculoides Anders.; Alta. (1).

S. balsamifera (Hook.) Barratt; Alta. (1).

S. glaucops Anders.; Alta. (1).

S. petrophila Rydb.; Alta., B.C. (1).

Salix spp.; Alta. (1).

Melampsora bigelowii Thuem.

O-I On Larix laricina (DuRoi) Koch.; Alta. (1).

L. lyallii Parl.; Alta. (1).

II-III On Salix amygdaloides Anders.; Man. (2).

Salix spp.; Sask., Alta. (25), Man. (19), B.C. (5).

Melampsora farlowii (Arth.) J. J. Davis

III On Tsuga canadensis (L.) Carr.; N.S. (1).

Melampsora lini (Pers.) Lév.

O-I-II-III Common on Linum spp.

Melampsora medusae Thuem.

O-I On Larix laricina (DuRoi) Koch.; N.S. (1).

II-III On Populus balsamifera L. (P. tacamahacca Mill.); Man. (19), Sask. (25), Nfld. (1).

$P$. deltoides Marsh.; Man. (25).

$P$. sargentii Dode.; Alta. (25).

P. tremuloides Michx.; Man. (19), Ont. (24).

Populus spp.; Man., Sask., Alta. (25), Ont. (7), Que. (14).

Melampsora occidentalis Jacks.

O-I On Pseudotsuga taxifolia (Poir.) Britt.; B.C., Alta. (37).

II-III On Populus acuminata Rydb.; B.C. (1).

P. balsamifera L.; B.C. (1), Sask. (25).

P. trichocarpa T. \& G.; B.C. (1, 37), Alta. (13).

Melampsora ribesii-purpurea Kleb.

O-I On Grossularia divaricata (Dougl.) Cov. \& Britt.; B.C. (1).

Ribes echinatum Lindl.; B.C. (1).

II-III On Salix bebbiana Sarg.; Alta. (24) 
Melampsorella cerastii (Pers.) Schroet.

O-I On Abies balsamea (L.) Mill.; Man., N.B., N.S., Ont., P.E.I., Nfld. (1), Que. (5).

A. grandis Lindl.; B.C. (1).

A. lasiocarpa (Hook.) Nutt.; B.C. (1).

Picea engelmanni (Parry) Engelm.; Sask. (1).

$P$. excelsa (Lam.) Link; Man. (1).

P. glauca (Moench) Voss (P. canadensis (Mill.) B.S.P.); Alta., Sask., Yukon (1), Man., Sask. (2).

P. glauca var. albertiana (S. Brown) Sarg.; B.C. (1).

P. mariana (Mill.) B.S.P.; Alta., Que., Nfld. (1), Man., Sask. (2).

II-III On Cerastium alpinum L.; B.C. (1).

C. arvense L.; Man., Sask. (25), Alta. (1).

Cerastium spp.; Man., B.C. (1).

Stellaria graminea L.; Ont. (24).

S. longipes Goldie; Alta. (1).

Melampsoridium betulinum (Pers.) Kleb.

II-III On Betula populifolia Marsh.; N.S. (1).

B. pumila L.; Nfld. (1).

Betula sp.; Man. (25), Alta. (11).

Milesia darkeri Faull

II-III On Cryptogramma acrostichoides R.BR.; B.C. (1).

Milesia fructuosa Faull

O-I On Abies balsamea (L.) Mill.; N.S., Ont., Que. (1).

II-III On Dryopteris intermedia (Muhl.) Gray; N.S., Que., Ont. (1).

D. spinulosa (O. F. Muell.) Watt.; Que. (1).

Milesia marginalis Faull \& Wats.

O-I On Abies balsamea (L.) Mill.; Ont. (1).

II-III On Dryopteris marginalis (L.) Gray.; Ont., Que. (1).

Milesia polypodophila (Bell) Faull

O-I On Abies balsamea (L.) Mill.; N.S., Ont., Que. (1).

II-III On Polypodium virginianum L.; N.S., Ont., Que. (1).

Nyssopsora clavellosa (Berk.) Arth.

III On Aralia nudicaulis L.; N.S., N.B., Ont., Que., Nfld. (1), Sask., Man. (2).

Phragmidium americanum (Peck) Diet.

O-I-II-III On Rosa blanda Ait.; Ont. (1).

$R$. virginiana Mill.; N.S., Ont. (1).

Rosa spp.; Ont. (1), Que. (14), N.B., N.S. (23).

"Phragmidium andersoni Shear

I-II-III On Dasiphora fruticosa (L.) Rydb. (Potentilla f. L.); Alta., B.C.,

N.B., Ont. (1), Man., Sask. (2), Que. (14).

Phragmidium disciflorum (Tode) J. F. James

(legitimate name, $P$. mucronatum (Pers.) Schlecht.)

O-I-II-III On Rosa spp., N.B. (1), B.C., Que., N.S. (23), Ont. (14), Man. (25). Phragmidium subcorticinum (Schrank) Wint.

Reported from Kentville N.S. (14), Ont. (20). On Rosa spp. Spore stage in doubt. 
Phragmidium ivesiae Syd.

I-II-III On Potentilla bipinnatifida Dougl.; Man. (2).

P. muttallii Lehm.; Alta. (1), Sask. (2).

Potentilla spp.; Alta. (1), Alta., Sask. (25).

Phragmidium montivagum Arth.

O-I-II-III On Rosa blanda Ait.; Man. (25).

R. bourgeauiana Crépin; Alta. (1).

Rosa sp.; Man. (25).

Phragmidium occidentale Arth.

O-I-II-III On Rubus parviflorous Nutt.; B.C. (1).

Phragmidium potentillae (Pers.) Karst.

O-I-II-III On Potentilla spp.; N.W.T., Alta., Man., Sask., Nfld. (1).

Phragmidium rosae-acicularis Liro

O-I-II-III On Rosa spp.; Alta., B.C., Ont., Man. (1).

Phragmidium rosae-arkansanae Diet.

O-I-II-III On Rosa suffulta Greene; Alta. (1).

II-III On Rosa sp.; Sask., Alta. (25).

Phragmidium rosae-californicae Diet.

O-I-II-III On Rosa spp.; B.C. (1).

Phragmidium rosae-pimpinellifoliae Diet.

O-I-II-III On Rosa spp.; Que., P.E.I. (23).

Phragmidium rosicola (Ell. \& Ev.) Arth.

III On Rosa suffulta Greene; Alta. (1).

Rosa sp.; Sask. (1).

Phragmidium rubi-idaei (DC.) Karst.

O-I-II-III Orı Rubus spp.; Alta., B.C., Man., N.B., N.S., Ont., Sask., Que., Nfld. (1).

R. idaeus L. var. strigosus Maxim.; Man. (2).

$R$. occidentalis L.; Nfld. (1).

Phragmidium rubi-odorati Diet.

I-II-III On Rubus odoratus L.; Ont. (1).

Phragmidium speciosum (Fries) Cooke

O-I-III On Rosa blanda Ait.; N.S. (1).

R. macounii Greene; Sask. (2).

R. virginiana Mill.; N.S. (1).

Rosa spp.; Man., Sask., (25) Alta., Ont., Que., N.B., N.S. (23).

Pileolaria toxicodendri (Berk. \& Rav.) Arth.

O-II-III On Rhus aromatica Ait.; Ont. (1).

$R$. radicans L.; Man., Ont., Que. (1).

( $R$. toxicodendron auct.); Man. (2), Que. (13).

Puccinia absinthii (Hedw.f.) DC.

II-III On Artemesia cana Pursh; Sask., Alta. (25).

A. frigida Willd.; Sask., Alta. (25), Man. (6).

A. gnaphalodes Nutt.; Man. (2).

A. ludoviciana Nutt.; B.C. (1).

Puccinia adoxae (Hedw.f.) DC.

III On Adoxa sp.; Alta. (26). 
Puccinia albulensis Magn.

III On Veronica alpina L. var. unalaschcensis C. \& S. (V. wormskjoldii R. \& S.); B.C. (1).

Puccinia amphigena Diet.

O-I On Maianthemum canadense Desf.; Sask. (2).

Nemexia lasioneuron (Hook.) Rydb.; Man., Sask. (25).

Smilacina stellata (L.) Desf.; Sask. (25)

Smilax herbacea L.; Man. (2).

II-III On Calamovilfa longifolia (Hook.) Hack.; Man. (2).

Puccinia andropogonis Schw.

$P$. andropogonis var. micropunctata Arth.

O-I On Castilleja sessiliflora Pursh; Sask. (2).

$P$. andropogonis var. melampyri Arth.

O-I On Melampyrum lineare Lam.; Ont. (1).

$P$. andropogonis var. onobrychidis Arth.

O-I On Lupinus perennis L.; N.S. (1).

Petalostemon candidus (Willd.) Michx.; Man. (2).

$P$. purpureus (Vent.) Rydb.; Man. (2).

$P$. andropogonis var. pentstemonis Arth.

O-I On Pentstemon acuminatus Dougl.; Sask. (1), Man. (2).

$P$. albidus Nutt.; Sask. (2).

$P$. eriantherus Pursh; Sask. (2).

$P$. nitidus Dougl.; Sask. (2).

$P$. andropogonis var. polygalina Arth.

O-I On Polygala senega L.; Man. (2).

$P$. andropogonis var. pustulata Arth.

O-I On Comandra pallida A. DC.; Man. (2).

C. umbellata (L.) Nutt.; Ont. (1), Man. (2).

II-III On Andropogon provincialis Lam.; Ont. (1).

A. scoparius Michx.; Man., Sask. (2).

Puccinia anemones-virginianae Schw.

III On Anemone canadensis L.; Man., Sask. (2).

A. riparia Fern.; Man. (16).

A. virginiana L.; Ont., Que. (1).

Puccinia angelicae (Schum.) Fuckel

II-III On Zizia aurea (L.) Koch; Man. (25).

Puccinia angustata Peck

O-I On Lycopus americanus Muhl.; Ont.(1).

L. asper Greene; Sask. (25).

L. rubellus Moench; Ont. (1).

Lycopus spp.; N.B., Ont. (1).

Mentha arvensis L.; N.S. (1).

$M$. canadensis L.; Man. (1).

M. glabrior (Hook.) Rydb.; Man. (25).

Senecio triangularis Hook.; B.C. (1).

II-III On Eriophorum angustifolium Roth.; Sask. (25).

E. viridicarinatum (Engelm.) Fern.; B.C. (1).

Scirpus atrovirens Muhl.; Ont., Que. (1), Man. (25).

S. cyperinus (L.) Kunth.; N.S., Que., Ont. (1).

S. microcarpus Presl; Man. (25), Sask. (2). 
Puccinia antirrhini Diet. \& Holw.

II-III On Antirrhinum majus L.; all provinces except Nfld. (23).

Puccinia arabicola Ell. \& Ev.

O-I-III On Cardamine douglasii (Torr.) Britt.; Ont. (1).

Puccinia arenariae (Schum.) Wint.

III On Honkenya peploides (L.) Ehrh.; N.S. (1).

Merckia physodes Fisch.; N.W.T. (1).

Moehringia lateriflora (L.) Fenzl; Sask. (2).

Stellaria longifolia Muhl.; Sask. (2).

S. longipes Goldie; Alta. (1), Sask. (2).

Puccinia areolata Diet. \& Holw.

O-I-II-III On Caltha leptosepala DC.; B.C., Sask. (1).

Puccinia argentata (Schultz) Wint.

O-I On Adoxa sp.; Alta. (26).

II-III On Impatiens biflora Walt.; Sask. (2).

I. pallida Nutt.; Alta. (1).

I. noli-tangere L.; Alta. (26).

Puccinia aristidae Tracy

O-I On Triglochin maritima L.; Alta. (1), Sask. (2).

Polygonum erectum L.; Sask. (2).

P. neglectum Bess.; Sask. (2).

Chenopodium album L.; Alta. (1), Man., Sask. (2).

Sarcobatus vermiculatus (Hook.) Torr.; Sask. (2).

Spinacia oleracea L.; Alta. (1).

Cheirinia cheiranthoides (L.) Link; Sask. (2).

Draba lutea Gilib.; Alta. (1).

Sisymbrium altissimum L.; Sask. (2).

Thlaspi arvense L.; Sask. (2).

Glaux maritima L.; Sask. (1)

Lappula echinata Gilib.; Man. (2).

Plantago eriopoda Torr.; Sask., Alta. (2).

II-III On Distichlis spicata (L.) Greene; Alta. (1), Man., Sask.". (2).

Puccinia asarina Kunze

III On Asarum caudatum Lindl.; B.C. (1).

Puccinia asparagi DC.

O-I-II-III On Asparagus officinalis L.; Man., Sask. (2), B.C. (12), Que. (11), Ont. (13), N.S. (10), N.B. (5), P.E.I. (3).

Puccinia asteris Duby

III On Aster cordifolius L.; Ont. (2).

A. ericoides L.; Sask. (2).

A. laevis L.; Sask. (25).

A. lindleyanus T. \& G.; Man. (2).

A. novae-angliae L.; Man. (25) Ont. (14).

Psilactis asteroides Gray; Nfld. to B.C. (1).

Puccinia atrofusca (Dudl. \& Thomp.) Holw.

O-I On Artemesia biennis Willd.; Alta. (1).

A. camporum Rydb.; Alta. (1), Sask. (2).

A. caudata Michx.; Alta. (1).

A. frigida Willd.; Alta. (1). 
A. glauca Pall.; Sask. (2).

A. gnaphalodes Nutt.; Man., Sask., Alta. (2).

A. purshiana Bess.; Alta. (1), Sask. (2).

II-III On Carex douglasii Boott; Alta. (1), Man. (2).

C. eleocharis Bailey; Yukon (1).

C. filifolia Nutt.; Alta. (1), Sask. (2).

C. praegracilis Boott; Man. (2).

C. rossii Boott; B.C. (1).

Puccinia atropuncta Peck \& Clint.

II-III On Zygadenus chloranthus Richards.; Sask. (2), Ont. (1).

Puccinia balsamorhizae Peck

O-I-II-III On Balsamorrhiza sagittata (Pursh) Nutt.; B.C. (1.)

Puccinia bardanae (Wallr.) Cda.

O-I-II-III On Arctium minus Schk.; N.S., Ont. (1), Man. (2).

Puccinia bistortae (Strauss) DC.

II-III On Bistorta vivipara (L.) S. F. Gray; Alta., Ont. (1), Que. (6).

Puccinia blasdalei Diet. \& Holw.

O-I-II-III On Allium textile N. \& M.; Alta. (1).

Puccinia bolleyana Sacc.

II-III On Carex intumescens Rudge; N.S. (1).

C. lupuliformis Sartw.; Ont. (1).

Puccinia calthae (Grev.) Link

O-I-II-III On Caltha palustris L.; Man. (1), Ont. (1).

Puccinia calthaecola Schroet.

O-I-II-III On Caltha palustris L.; Man. (1), Sask. (2).

Puccinia campanulae Carm.

III On Campanula rotundifolia L. var. intercedens Witasek.; Que. (17)

Puccinia canadensis Arth.

III On Viola orbiculata Geyer.; Alta., B.C. (1).

Puccinia canaliculata (Schw.) Lagerh.

II-III On Cyprus strigosus L.; Ont. (1).

Puccinia caricis (Schum.) Schroet.

(legitimate name, $P$. caracina DC.)

$P$. caricis var. urticata (Kern) Arth.

O-I On Urtica dioica L.; Ont. (1).

$U$. gracilis Ait.; Ont. (1), Man. (2).

U. lyallii Wats.; B.C. (1), Sask. (2).

II-III On Carex vesicaria L.; Man., Sask. (2).

Carex spp.; Man., Sask. (2).

$P$. caricis var. grossulariata Arth.

O-I On Grossularia cynosbati (L.) Mill.; Ont., Que. (1).

G. hirtella (Michx.) Spack; N.S. (1).

G. setosa (Lindl.) Cov. \& Britt.; Alta. (1).

Ribes americanum Mill.; Ont. (1), Man., Sask. (2).

$R$. bracteosum Dougl.; B.C. (1).

R. glandulosum Grauer; Alta., B.C., N.S., Labr. (1).

R. grossularia L. (Grossularia uva-crispa (L.) Mill.); Man., Sask. (2).

$72559-3 \frac{1}{2}$ 
R. hudsonianum Richards.; Alta. (1).

$R$. lacustre (Pers.) Poir.; Alta., Ont. (1).

$R$. laxiflorum Pursh; B.C. (1).

R. oxycanthoides L. (G. o. (L.) Mill.); Man., Sask. (2).

$R$. nigrum L.; Sask. (2).

$R$. setosum Lindl.; Sask. (2).

Ribes spp.; N.B., P.E.I. (23).

II-III On Carex spp.; Man., Sask. (2).

$P$. caricis var. uniporula (Orton) Arth.

O-I On Grossularia cynosbati (L.) Mill.; Ont. (1).

II-III On Carex spp.; Nfld., N.S., Que., Ont. (1).

Puccinia caricis-shepherdiae J. J. Davis

O-I On Elaeagus angustifolia L.; Sask. (1), Man. (16). B.C. (8).

E. commutata Bernh.; Alta., Man., Sask. (2).

Shepherdia argentea Nutt.; Sask. (2).

S. canadensis (L.) Nutt.; Man., Sask. (2), Alta., Ont., Nfld. (1).

II-III On Carex aquatilis Wahl.; Sask. (1).

C. atherodes Spreng.; Sask. (2).

C. eburnea Boott; Ont., Que. (1).

C. lanuginosa Michx.; Sask. (2).

C. substricta (Kuekenth.) Mack.; Sask. (2).

C. vesicaria L., Sask. (2).

Puccinia carthami Corda

O-II-III On Carthamus tinctorius L.; Alta., Man. (14), Sask. (12).

Puccinia cicutae Lasch

O-I-II-III On Cicuta maculata L.; N.S., Man. (1).

C. occidentalis Greene; Sask. (2).

Puccinia circaeae Pers.

III On Circaea alpina L.; Nfld., N.S., Ont., Sask. (1), Man. (2) Que. (5).

C. latifolia Mill.; N.S., Que., Ont (1).

C. pacifica Asch. \& Magn.; B.C. (1).

Puccinia cirsii Lasch

O-I-II-III On Cirsium flodmanii (Rydb.) Arth.; Man., Sask. (2).

C. undulatum (Nutt.) Spreng.; Alta. (1), Man. (2).

Cirsium spp.; Sask. (2).

Puccinia cnici Mart.

O-I-II-III On Cirsium lanceolatum (L.) Hill; B.C., Ont. (1).

Puccinia cockerelliana Bethel

II-III On Festuca scabrella Torr.; Alta. (1).

Puccinia comandrae Peck

II-III On Comandra livida Richards.; Man. (2).

C. pallida A. DC.; Sask. (2).

Puccinia commutata Syd.

O-I-III On Valeriana septentrionalis Rydb.; Alta. (1).

$V$. sitchensis Bong.; B.C. (1).

Puccinia conglomerata (Strauss) Schmidt \& Kunze

III On Petasites frigidus (L.) Fries; Alta., B.C. (1).

P. palmatus (Ait.) Gray; Alta., Ont., Sask. (1), Man. (2). 
Puccinia convolvuli (Pers.) Cast.

O-I-II-III On Convolvulus sepium L.; Que. (1), Man. (2).

Puccinia coronata Corda

O-I On Elaeagnus commutata Bernh.; Man., Sask. (25).

Rhamnus alnifolia L'Her.; Man., Sask., Ont. (1), Que. (17).

R. cathartica L.; Sask., Ont. (1), Man. (2), B.C., Que., N.B., N.S., P.E.I. (23).

R. davurica Pall.; Man. (22).

R. frangula L.; N.B. (18).

$R$. purshiana DC.; B.C. (1).

R. utilis Decne.; Man. (22).

Shepherdia canadensis (L.) Nutt.; Alta., Yukon, Sask., Man. (1).

II-III Common on oats and many cultivated and native grasses. Varieties of this rust species have been separated in Europe and North America.

The varieties of this rust found in Canada are:

$P$. coronata var. avenae Erikss.

O-I On Rhamnus cathartica L. (1).

II-III On Avena fatua L. (1).

Avena spp., (1).

Lamarkia aurea (L.) Moench (Achyrodes aureum Ktze.) (1).

$P$. coronata var. calamagrostis Fraser \& Led.

O-I On Rhamnus alnifolia L'Her. (1).

II-III On Calamagrostis canadensis (Michx.) Beauv. (1).

C. elongata (Kearney) Rydb. (1).

Scolochloa festucacea Link (1).

P. coronata var. bromi Fraser \& Led.

O-I On Shepherdia canadensis (L.) Nutt. (1)

II-III On Bromus ciliatus L. (1).

B. latiglumis (Shear) Hitchc. (1).

B. pumpellianus Scribn. (1).

$P$. coronata var. elaeagni Fraser \& Led.

(O)-I On Elaeagnus commutata Bernh. (1).

II-III On Calamagrostis montanensis Scribn. (1).

(Besides the varieties reported above, var. agrostis Erikss. has been isolated from aecia on Rhamnus frangula from N.B., var. festucae Erikss. from aecia on $R$. cathartica from Eastern Canada, and an apparently new variety (20), subsequently named $P$. coronata f.sp. secalis by B. Peturson (Can. J. Botany 32:42 1954), from aecia on $R$. cathartica from Man. and Eastern Canada; II-III on Agropyron repens (L.) Beauv.; capable of attacking other wild grasses and cultivated varieties of Hordeum vulgare and Secale cereale).

Puccinia crandallii Pamm. \& Hume

O-I On Symphoricarpos albus (L.) Blake; B.C. (1).

S. occidentalis Hook.; Sask. (1), Man. (2).

Symphoricarpos spp.; Alta. (23).

II-III On Festuca ovina L.; Sask. (2).

F. saximontana Rydb.; Sask. (1). 
Puccinia cruciferarum Rud.

III On Cardamine bellidifolia L.; B.C. (1).

Puccinia cryptotaeniae Peck

O-III On Cryptotaenia canadensis (L.) DC.; Que. (1).

Puccinia cyani (Schleich.) Pass.

O-I-III On Centaurea cyanus L.; Ont. (1), B.C. (16), N.S. (23).

Puccinia cypripedii Arth. \& Holw.

II On Cypripedium parviflorum Salisb.; Sask. (1).

Puccinia dayi G. W. Clint.

III On Steironema ciliatum (L.) Raf.; Ont., Alta., Sask. (1).

Puccinia difformis Kunze

O-I-III On Galium aparine L.; B.C. (1).

Puccinia distichlidis Ell. \& Ev.

O-I On Glaux maritima L.; Sask. (25).

Steironema ciliatum (L.) Raf.; Man., Sask. (25).

II-III On Spartina gracilis Trin.; Sask., Man. (25).

S. pectinata Link (S. michauxiana Hitchc.); Man. (1), Sask., (25).

Spartina sp.; Sask. (25).

Puccinia douglasii E. \& E.

O-I-III On Phlox hoodii Richards.; Alta., Sask. (1).

Puccinia drabae Rud.

III On Draba arabisans Michx.; Que. (1).

D. incerta Payson; Alta. (1).

Draba sp.; Man. (2).

Puccinia eatoniae Arth.

$P$. eatoniae var. ranunculi Mains

O-I On Ranunculus abortivus L.; Ont. (1), Man. (2).

II-III On Sphenopholis obtusata (Michx.) Scribn.; Sask. (1).

S. pallens (Spreng.) Scribn.; Sask. (1).

Puccinia eleocharidis Arth.

O-I On Eupatorium perfoliatum L.; N.S., Ont. (1).

E. purpureum L.; N.S., Ont. (1).

E. purpureum var. maculatum (L.) Darl.; Man. (2).

II-III On Eleocharis palustris (L.) R. \& S.; Ont., Que. (1). Eleocharis spp.; Man. (2).

Puccinia ellisiana Thuem.

O-I On Viola nephrophylla Greene.; Sask. (25).

Viola sp.; Man., Sask. (25).

II-III On Andropogon provincialis Lam.; Sask. (25), Man. (6).

Schizachyrium scoparium (Michx.) Nash; Sask. (25).

Puccinia emaculata Schw.

II-III On Panicum capillare L.; Ont. (1).

Puccinia epilobii DC.

III On Epilobium alpinum L.; Que. (1).

Epilobium sp.; Sask. (25).

Puccinia erigeniae (Orton) Arth.

O-I-III On Erigenia bulbosa (Michx.) Nutt.; Ont. (1). 
Puccinia extensicola Plowr.

(legitimate name, $P$. dioicae P. Magn.)

$P$. extensicola var. asteris Arth.

I On Aster spp.; Man., Sask. (2).

II-III On Carex spp.; Man., Sask. (2).

$P$. extensicola var. erigerontis Arth.

II-III On Carex spp.; B.C. and east of the Rocky Mts. (1).

$P$. extensicola var. solidaginis Arth.

I On Solidago nemoralis Ait.; Man. (2).

S. rigida L.; Man. (2).

S. serotina Ait.; Sask., Man. (2).

II-III On Carex spp.; Man., Sask. (2).

$P$. extensicola var. euthamii Arth.

I On Euthamia graminifolia (L.) Nutt. (Solidago g. (L.) Salisb.); Ont. (1).

II-III On Carex scoparia Schk.; N.S., Ont., Que. (1).

$P$. extensicola var. oenotherae Arth.

I On Chamaenerion spicatum (Lam.) S. F. Gray; Sask., Man. (1).

Oenothera biennis L.; Man., Sask. (2).

II-III On Carex sartwelli Dewey; Man. (2).

$P$. extensicola var. hieraciata Arth.

I On Senecio columbianus Greene; Sask. (1).

S. flavulus Greene; Alta. (1).

Hieracium scabriusculum Schw.; Man., Sask. (2).

Lactuca pulchella (Pursh) DC.; Man., Sask. (2).

L. sativa L.; Man., Sask. (2), Alta., Ont., Que., N.B., N.S. (23).

Lygodesmia juncea (Pursh) D. Don; Sask. (2).

Nabalus albus (L.) Hook.; Man. (2).

Agoseris glauca (Nutt.) Greene; Sask. (2).

II-III On Carex sprengelii Dewey; Sask. (2).

Carex spp.; Man., Sask. (2).

$P$. extensicola var. phrymae Arth.

II-III On Carex sprengelii Dewey; Ont. (1).

Puccinia fergussoni Berk. \& Br.

III On Viola langsdorfii Fisch.; B.C. (1).

V. mackloskeyi Lloyd; B.C. (1).

V. nephrophylla Greene; B.C. (1).

Puccinia festucae (DC.) Plowr.

O-I On Lonicera caerulea L.; N.B. (1).

Puccinia fischeri Cruchet \& Mayor

III On Saxifraga oppositifolia L.; Yukon (34).

Puccinia fraseri Arth.

III On Hieracium scabrum Michx.; N.S., Que. (1).

Puccinia gentianae (Str.) Link

O-I-II-III On Gentiana affinis Griesb.; Sask. (1).

G. andrewsii Griesb.; Ont. (1).

G. interrupta Greene; Sask. (1). 
Puccinia gigantea Karst.

III On Chamaenerion spicatum (Lam.) S. F. Gray; Alta., Man. (1).

Puccinia gigantispora Bubak

O-I-III On Anemone cylindrica Gray; Alta., B.C., Sask. (1).

A. globosa Nutt.; Alta., B.C. (1).

Puccinia glumarum (Schmidt.) Erikss. \& Henn.

II-III Common on graminaceous hosts in southwestern Sask., in Alta. and B.C.

Puccinia graminis Pers.

O-I On Berberis vulgaris L.; wherever this host occurs.

B. aquifolium Pursh, (Mahonia a. (Pursh) Nutt.); Sask. (2). Berberis spp.; Que. (16), Ont. (6).

Mahonia sp.; Ont. (6).

II-III Common on graminaceous hosts.

The following varieties of this rust occur in Canada:-

$P$. graminis var. avenae Erikss. \& Henn.

II-III On Avena spp.; common.

$P$. graminis var. poae Erikss. \& Henn.

II-III On Poa spp.; Man. (6).

P. graminis var. phlei-pratensis (Erikss. \& Henn.) Stakm. \& Piem.

II-III On Phleum pratense L.; common.

Brachypodium pinnatum Beauv.; Man. (16).

P. graminis var. secalis Erikss. \& Henn.

II-III On Secale cereale L.; common.

Hordeum vulgare L.; fairly common.

$P$. graminis var. tritici Erikss. \& Henn.

II-III On Triticum spp.; common.

Agropyron spp.; common.

Hordeum jubatum L.; common.

Hordeum vulgare L.; common.

(To the varieties mentioned above may be added $P$. graminis var. agrostidis Erikss., which T. Johnson and G. J. Green (Can. J. Botany 29: 1-9. 1951) found to be the second most common variety isolated from aecia on naturally infected barberry in eastern Canada).

Puccinia granulispora Ell. \& Gall.

O-I-II-III On Allium cernuum Roth; Alta, B.C. (1). Allium sp.; B.C. (1).

Puccinia grindeliae Peck

III On Grindelia perennis A. Nels.; Alta., Sask. (2).

G. squarrosa (Pursh) Dunal.; Alta., Sask. (25).

Lygodesmia juncea (Pursh) D. Don.; Man. (25).

Sideranthus spinulosus (Pursh) Sweet; Sask. (25).

Puccinia grumosa Syd. \& Holw.

O-I-II-III On Zygadenus elegans Pursh; B.C. (1).

Puccinia haleniae Arth. \& Holw.

III On Gentiana amarella L. var. acuta (Michx.) Herder; Ont. (2). 
Puccinia helianthi Schw.

O-I-II-III On Helianthus annuus L.; cult. common.

H. aridus Rydb.; Sask. (2).

H. fascicularis Greene; Man. (1), Sask. (2), Alta. (14).

H. giganteus L.; Ont. (1), Sask. (25).

H. maximiliani Schrad.; Man. (25).

$H$. rigidus (Cass.) Desf.; Ont. (1).

H. strumosus L.; Ont. (1).

H. subrhomboideus Rydb.; Sask. (25).

H. subtuberosus Bourg.; Alta. (2).

H. tuberosus L.; Ont. (1), Man. (2), N.S. (23).

Puccinia heucherae (Schw.) Diet.

$P$. heucherae var. austroberingiana Savile

III On Mitella breweri Gray; B.C. $(1,34)$.

M. trifida Graham; B.C. (34).

Saxifraga punctata sspp.; B.C., Yukon, Keew. (34).

Tellima grandiflora (Pursh) Dougl.; B.C. $(1,34)$.

$P$. heucherae var. heucherae

III On Heuchera cylindrica Dougl. var. glabella (T. \& G.) Wheelock; B.C. (34).

H. glabra Willd.; B.C. $(1,34)$.

H. micrantha Dougl. ex. Lindl. vars.; B.C. (34).

H. ovalifolia Nutt.; Alta. (1), B.C. (34).

Mitella diphylla L.; Ont. (1).

M. nuda L.; Alta., Sask., Ont. (1), Man. (2), B.C., Mack., Que., Nfld. (34).

$M$. ovalis Greene; B.C. (34).

Saxifraga lyallii Engler (Micranthes l. (Engler) Small); B.C. (34), Alta. (1, 34).

Tiarella cordifolia L.; Ont. (1, 34), Que. (1).

T. unifoliata Hook.; B.C. $(1,34)$.

Tolmiea menziesii (Pursh) T. \& G.; B.C. (34).

$P$. heucherae var. saxifragae (Schlecht.) Savile

III On Heuchera cylindrica Dougl. var. trionalis R.B. \& L.; B.C., Alta. (34).

H. ovalifolia Nutt.; B.C. (34).

$H$. richardsonii $\mathrm{R} . \mathrm{Br}$.; Man. $(1,34)$.

Saxifraga cernua L.; Mack., Frank., Keew., Que. (34).

S. hieracifolia Waldst. \& Kit.; Frank., Keew. (34).

S. nivalis L.; Frank., Keew. (34).

S. occidentalis S. Wats. ssp. rufidula (Small) Basigalupi; B.C. (34).

S. rivularis L.; B.C. (1, 34), Frank., Que., Labr. (34).

S. tenuis (Wallr.) Sm.; Frank. (34).

S. virginiensis Michx.; Que. (34).

Puccinia hieracii (Schum.) Mart.

O-I-II-III On Agoseris glauca (Nutt.) Greene; Sask. (2).

Apargia autumnalis (L.) Hoffm.; N.S. (1).

Cichorium intybus L.; Ont., Que., N.S. (1).

Crepis runcinata (James) T. \& G.; Sask. (2). 
Hieracium canadense Michx.; Alta., Ont., Que. (1), Man. (2).

H. scabriusculum Schw.; Sask., Man. (2).

H. scabrum Michx.; Man. (2), N.S. (1).

Taraxacum dumetorum Greene; Sask. (2).

T. kok-saghyz Rod.; Alta., Man., Ont., N.S. (14).

T. vulgare (Lam.) Schr.; B.C., Alta., Sask., Ont., Que., N.S. (1), Man. (2).

Puccinia holboellii (Hornem.) Rostr.

O-III On Arabis divaricarpa Nels.; Alta. (1).

A. drummondii A. Gray; B.C. (1).

A. lignipes A. Nels.; Alta. (1).

A. lyallii Wats.; B.C. (1).

Puccinia hordei Otth

II-III Common (23). On cultivated barley in most provinces, particularly Ont. and Que.

Puccinia intermixta Peck

O-I-III On Iva axillaris Pursh; Sask., Man. (2).

Puccinia investita Schw.

O-I-III On Gnaphatium macounii Greene; Ont. (1).

Puccinia iridis (DC.) Wallr.

II-III On Iris versicolor L.; N.S., Ont., Que. (1), Man. (2).

Iris sp.; N.B., P.E.I. (4), Que. (15).

Puccinia karelica Tranz.

I On Trientalis americana Pursh; N.S. (1).

II-III On Carex paupercula Michx.; N.S. (1).

Puccinia koeleriae Arth.

O-I On Mahonia aquifolium (Pursh) Nutt.; B.C. (1).

$M$. repens (Lindl.) Don.; B.C. (1).

II-III On Koeleria cristata (L.) Pers.; Sask. (25).

Puccinia lapsanae (Cooke) Fckl.

O-I-II-III On Lapsana communis L.; Ont. (1).

Puccinia liatridis (Webber) Bethel

O-I On Liatris aspera (Michx.) Greene; Man., Sask. (2).

L. ligulistylis (A. Nels.) Rydb.; Sask. (2).

L. punctata Hook.; Sask. (2).

II-III On Agrostis hyemalis (Walt.) B.S.P.; Man., Sask. (2).

Puccinia ligustici Ell. \& Ev.

III On Ligusticum apiifolium (Nutt.) Gray; B.C. (1).

Puccinia limosae Magn.

O-I On Naumburgia thyrsiflora (L.) Duby; Ont., Man. (1).

II-III On Carex limosa L.; Ont. (1).

C. stratiformis Britt.; Que. (1).

Puccinia linkii Klotzsch

III On Viburnum pauciflorum Pylaie; Man., Sask., Que. (1).

Puccinia lobeliae Ger.

III On Lobelia syphilitica L.; Ont. (1).

Puccinia maculosa Schw.

III On Agoseris glauca (Nutt.) Greene; Alta. (1).

Nabalus racemosus (Michx.) DC.; Que. (1). 
Puccinia magnusiana Koern.

O-I On Anemone canadensis L.; Sask. (1), Man. (2).

II-III On Phragmites communis Trin.; Man. (2).

Puccinia malvacearum Bert.

III On Althaea ficifolia (L.) Cav.; Ont. (1).

A. rosea (L.) Cav.; common.

Malva moschata L.; Ont. (1).

M. rotundifolia L.; Ont. (1), Man. (2), Que. (6).

Malva spp.; Man., Que., Ont. (23).

Puccinia mariae-wilsoni $\mathrm{G}$. W. Clint.

O-I-III On Claytonia caroliniana Michx.; Ont. (1).

C. virginica L.; Ont., Que. (1).

Puccinia marylandica Lindr.

O-I-II-II On Sanicula marilandica L.; Alta., Sask., Man. (1).

Sanicula sp.; N.S. (1).

Pucinia mcclatchieana Diet. \& Holw.

II-III On Scirpus microcarpus Presl; B.C., N.S. (1).

Puccinia menthae Pers.

O-I-II-III On Clinopodium vulgare L.; Ont. (1).

Mentha arvensis L.; N.S. (1).

M. canadensis L.; N.S., Que., Ont., Sask., Alta. (6), B.C. (5).

M. glabrior (Hook.) Rydb.; Man., Sask. (2).

M. piperta L.; Man. (15).

M. spicata L.; Ont. (1), B.C. (22).

Monarda didyma L.; Ont. (1).

M. fistulosa L.; Alta., Ont., Man. (1).

M. menthaefolia Benth.; Alta. (1), Sask. (2).

M. mollis L.; Alta., Man., Yukon (1).

M. mollis var. menthaefolia Fern.; B.C. (6).

Pycnanthemum flexuosum (Walt.) B.S.P.; Ont. (1).

P. virginianum (L.) Dur. \& Jacks.; Ont. (1).

Puccinia mesomajalis Berk. \& Curt.

III On Clintonia borealis (Ait.) Raf.; N.S. (1), Ont. (2).

C. uniflora (Schult.) Kunth; B.C. (1).

Puccinia microica Ellis

II-III On Cryptotaenia canadensis (L.) DC.; Que. (1).

Puccinia millefolii Fuckel

III On Achillea millefolium L.; Man., Sask. (2), Que. (20).

A. multiflora Hook.; Alta. (1).

A. ptarmica L.; Que. $(17,20)$.

Artemisia frigida Willd.; Man. (2), B.C. (1).

Puccinia minussensis Thuem.

O-I-II-III On Lactuca pulchella (Pursh) DC.; Man., Sask., Alta., B.C. (1).

Puccinia minutissima Arth.

O-I On Decodon verticillatus (L.) Ell.; Ont. (1).

II-III On Carex lasiocarpa Ehrh.; Ont. (1).

C. substricta (Kuek.) Mack.; Ont. (1).

$72559-4 \frac{1}{2}$ 
Puccinia monoica (Peck) Arth.

O-I On Arabis brachycarpa (T. \& G.) Britt.; Alta. (1), Sask. (2).

A. drummondii Gray; Alta. (1).

A. lyrata L.; Ont. (1).

A. nuttallii B. L. Rob.; B.C. (1).

A. ovata (Pursh) Poir.; Sask. (2).

A. retrofracta Grah.; B.C. (1), Sask. (2).

Arabis spp.; Man. (2).

II-III On Koeleria cristata (L.) Pers.; Sask. (2).

Trisetum spicatum (L.) Richt.; Sask. (2).

Puccinia montanensis Ellis

II-III On Agropyron trachycaulum (Link) Malte var. unilaterale (Cassidy)

Malte; Man. (16).

Agropyron spp.; Alta., Sask., Man. (1).

Elymus canadensis L.; Alta., Man. (1).

E. curvatus Piper; Man. (1).

E. dahuricus Turcz.; Man. (14).

Puccinia obscura Schroet.

II-III On Luzula campestris (L.) DC. var. multiflora (Ehrh.) Celak.; N.S. (1), Sask. (2).

L. carolinae Wats.; N.S., Ont. (1).

L. parviflora (Ehrh.) Devs.; B.C. (1)

Puccinia obtecta Peck

II-III On Scirpus validus Vahl.; Ont., Sask. (1).

Puccinia obtegens (Link) Tul.

O-I-II-III On Cirsium arvense (L.) Scop.; N.S., Ont., Que. (1), P.E.I., Sask. (24). Collected at Comox, B.C., May, 1949. A. M. Brown.

A perennial rust cultured in garden at Winnipeg from 1940 to 1945. Originating from urediospores brought from England by the late Dr. A. H. R. Buller. Rust was lost due to road construction 1946. A.M.B.

Puccinia oenotherae Vize

O-I-II-III On Epilobium minutum Lindl.; B.C. (1).

Puccinia onopordi Syd.

II-III On Onopordum acanthium L.; N.S. (1).

Puccinia orbicula Pk. \& G. W. Clint.

O-I-II-III On Nabalus altissimus (L.) Hook.; N.S. (1).

N. racemosus (Michx.) DC.; Sask. (2)。

N. trifoliolatus Cass.; Que. (1).

Nabalus sp.; Ont. (1).

Puccinia ornata Arth. \& Holw.

III On Rumex britannica L.; Sask. (1).

$R$. occidentalis S. Wats.; Man., Sask., Alta. (2).

Rumex sp.; Alta., Man. (1).

Puccinia ornatula Holw.

III On Viola canadensis L.; B.C. (1).

V. glabella Nutt.; B.C. (1).

V. rugulosa Greene; B.C. (1). 
Puccinia ortonii Jacks.

O-I-II-III On Dodecatheon jeffreyi Moore; B.C. (1).

D. pauciflorum (Durand) Greene; Sask. (2).

Puccinia oxyriae Fuckel

II-III On Oxyria digyna (L.) Hill., Alta., B.C. (1).

Puccinia parkerae Diet. \& Holw.

III On Grossularia aivaricata (Dougl.) Cov. \& Britt.; B.C. (1).

Ribes echinatum Lindl.; B.C. (1).

$R$. lacustre (Pers.) Poir.; Alta. (2).

Puccinia parnassiae Arth.

III On Parnassia fimbriata Banks; Alta., B.C. (1).

Puccinia pazschkei Diet.

$P$. pazschkei var. heterisiae (Jacks.) Savile

III On Saxifraga mertensiana Bong.; B.C. (34).

P. pazschkei var. jueliana (Diet.) Savile

III On Saxifraga aizoides L.; Frank. (34).

S. ferruginea Graham (S. nootkana Moq.); B.C. $(1,34)$.

P. pazschkei var. oppositifoliae Savile

III On Saxifraga oppositifolia L.; Que. (34).

$P$. pazschkei var. tricuspidatae Savile

III On Saxifraga bronchialis L.; B.C. (34).

S. tricuspidata Rottb. (Leptasea t. (Rottb.) Haw.); N.W.T.

(1), Yukon, Mack., Frank., Keew. (34).

Puccinia phragmitis (Schum.) Koern.

O-I On Rheum rhaponticum L.; Man. (2).

Rumex mexicanus Meisn.; Man. (1).

$R$. occidentalis S. Wats.; Man. (2).

II-III On Phragmites communis Trin.; Man. (1).

Puccinia physalidis Peck

III On Physalis heterophylla Nees; Ont., Man. (1).

$P$. virginiana Mill.; Man. (1).

Puccinia pimpinellae (Strauss) Mart.

O-I-II-III On Osmorrhiza claytoni (Michx.) Clarke; Ont., Que. (1).

O. longistylis (Torr.) DC.; Ont. (1), Man., Sask. (2).

Puccinia poae-nemoralis Otth ( $P$. poae-sudeticae (West.) Jörstad)

II-III On Poa annua L.; Alta. (1).

P. compressa L., B.C. (23).

P. crocata Michx.; Alta. (1).

$P$. interior Ryd.; Alta. (1).

$P$. nemoralis L.; Alta. (1).

$P$. palustris L.; Alta. (1), Sask. (2).

P. pratensis L.; Alta., Ont., Que., Man. (1), Sask. (2), B.C., P.E.I. (23).

Anthoxanthum odoratum L.; N.S. (1).

P. poae-sudeticae var. airae (Lagerh.) Arth.

II-III On Deschampsia caespitosa (L.) Beauv.; Alta. (1).

Puccinia poarum Niels.

O-I On Tussilago farfara L.; N.S. (1). 
Puccinia podophylli Schw.

O-I-III On Podophyllum peltatum L.; Ont. (1).

Puccinia polygoni-amphibii Pers.

P. polygoni-amphibii var. convolvuli (Alb. \& Schw.) Arth.

II-III On Polygonum convolvulus L.; Ont. (1), Man. (2).

$P$. polygoni-amphibii var. persicariae (Strauss) Arth.

O-I On Geranium maculatum L.; Ont. (1).

II-III On Polygonum coccineum Muhl.; Ont., Que. (1).

P. hartwrightii Gray; Alta., B.C., N.S., Man. (1), Sask. (2).

P. hydropiperoides Michx,; Ont. (1).

P. lapathifolium L.; Ont. (1).

P. muhlenbergii (Meissn.) S. Wats.; Ont., Que. (1), Man., Sask., Alta. (2).

$P$. pensylvanicum L.; Que. (1).

$P$. persicaria. L; (4).

Puccinia porphyrogenita Curt.

III On Cornus canadensis L.; Nfld., B.C., N S., Sask. (1), Man. (2), Que. (5), P.E.I. (6).

Puccinia porri (Sow.) Wint.

II-III On Allium sp.; B.C. (18).

Puccinia praegracilis Arth.

$P$. praegracilis var. praegracilis

I On Habenaria gracilis Wats.; B.C. $(1,29)$.

II-III On Agrostis thurberiana Hitchc.; B.C. (1).

$P$. praegracilis var. connersii (Savile) Savile

syn. P. connersii Savile

I On Habenaria dilatata (Pursh) Hook.; Que. (29).

II-III On Deschampsia atropurpurea (Wahl.) Scheele; Que. (29).

Puccinia pulsatillae Kalchbr.

III On Anemone parviflora Michx.; B.C., Que. (1).

A. patens L. var. wolfgangiana (Bess.) Koch; Sask. (2).

Pulsatilla ludoviciana (Nutt.) Heller; Alta., Sask. (1).

Trautvetteria grandis Nutt.; B.C. (1).

Puccinia punctata Link

O-I-II-III On Galium asprellum Michx.; N.S., Ont. (1).

G. trifidum L.; Man. (2).

$P$. punctata var. troglodytes (Lindr.) Arth.

II-III On Galium triflorum Michx.; Man. (2).

Puccinia pygmaea Erikss.

II-III On Oryzopsis asperifolia Michx.; Ont. (1).

Puccinia pyrolae Cooke

III On Polygala paucifolia Willd.; Ont. (1).

Puccinia ranunculi Blytt

III On Ranunculus suksdorfii Gray; B.C. (1).

Puccinia recedens Syd.

III On Senecio willingii Greenm.; Alta. (1).

Puccinia ribis DC.

III On Ribes triste Pall.; Man., Sask. (2). 
Puccinia rubefaciens Johans.

III On Galium boreale L.; Alta. (1), Man., Sask. (2).

Puccinia rubigo-vera (DC.) Wint.

P. rubigo-vera var. agropyri (Erikss.) Arth.

O-I On Thalictrum spp.; common:

II-III On Poaceae common.

P. rubigo-vera var. agropyrina (Erikss.) Arth.

O-I On Thalictrum spp.; common.

II-III On Poaceae common

P. rubigo-vera var. tritici (Erikss. \& Henn.) Carl.

O-I On Thalictrum dipterocarpum Franch. and

T. glaucum Desf.; in greenhouse at Winnipeg. A.M.B.

II-III On Triticum spp.; common.

Secale cereale L. Rosen rye in greenhouse at Winnipeg.

A.M.B.

P. rubigo-vera var. secalis (Erikss.) Carl.

II-III On Secale cereale L.; common.

P. rubigo-vera var. apocrypta (Ell. \& Tracy) Arth.

O-I On Phacelia heterophylla Pursh; B.C. (1).

$P$. rubigo-vera var. impatientis (Arth.) Mains

O-I On Impatiens biflora Walt.; Man., Ont., Que. (1).

I. pallida Nutt.; Ont. (1).

II-III On Agrostis perennans (Walt.) Tuck., N.S. (1).

Elymus virginicus L.; Ont., Que. (1).

Hordeum jubatum L.; Man. (1).

Puccinia rydbergii Garrett

III On Sedum stenopetalum Pursh; B.C. (1).

Puccinia scaber (Ell. \& Ev.) Barth.

II-III On Stipa viridula Trin.; Alta. (1), Man., Sask. (2).

Puccinia scandica Johans.

III On Epilobium sp.; B.C. (1).

Puccinia schedonnardi Kell. \& Swingle

II-III On Muhlenbergia cuspidata (Torr.) Rydb.; Man. (14).

Puccinia septentrionalis Juel

II-III On Bistorta vivipara (L.) S. F. Gray; Nfld. (1).

Puccinia sessilis Schneid.

O-I On Convallaria sp.; N.S. (9).

Maianthemum canadense Desf.; Man. (2).

Smilacina stellata (L.) Desf.; B.C., Ont. (1), Man., Sask. (2).

Iris versicolor L.; Que., Man. (1).

II-III On Phalaris arundinacea L.; Ont., Man. (1), Sask. (2).

Puccinia seymouriana Arth.

O-I On Cephalanthus occidentalis L.; Que. (1).

II-III On Spartina pectinata Link (S. michauxiana Hitchc.); Ont., Que. (1).

Pucciana sherardiana Koern.

O-III On Sphaeralcea coccinea (Nutt.) Rydb.; Alta., Sask., Man. (2). 
Puccinia sorghi Schw.

II-III On Zea mays L.; Man., Sask. (2), P.E.I. (16), N.S., Ont., Que., N.B. (23).

Puccinia sparganioides Ellis \& Barth.

O-I On Fraxinus americanus L., Que. (4), N.S. (19).

Fraximus pennsylvanica Marsh. var. lanceolata (Borkh.) Sarg.; Que. (16), Man. (15).

II-III On Spartina pectinata (Link; Que. (18).

Puccinia sporoboli Arth.

O-I On Lilium umbellatum Pursh; Sask. (2). Lilium sp.; Man. (10).

Puccinia stipae Arth.

O-I On Chrysopsis hirsutissima Greene; Sask. (2).

Solidago glaberrima Mart.; Alta. (1).

S. mollis Bartl.; Alta. (1).

Solidago sp.; Sask. (2).

Lygodesmia juncea (Pursh) D. Don; Sask., Man. (2).

II-III On Stipa comata Trin. \& Rupr.; Alta., Man. (1), Sask. (2).

S. spartea Trin.; Man., Sask. (2).

S. tweedyi Scribn. \& Vasey.; Alta. (1).

Puccinia subcircinata Ell. \& Ev.

O-I-III On Senecio triangularis Hook.; Alta. (1).

Puccinia symphoricarpi Harkn.

III On Symphoricarpos albus (L.) Blake; B.C. (1).

Puccinia treleasiana Paz.

III On Caltha leptosepala DC.; B.C. (1).

Puccinia uliginosa Juel

I On Parnassia palustris L.; Man. (2).

Puccinia vagans (DC.) Arth.

$P$. vagans var. epilobii-tetragoni $\mathrm{DC}$.

O-I-II-III On Epilobium adenocaulon Haussk.; Sask. (2).

Puccinia variabilis Grev.

O-II-III On Taraxacum vulgare (Lam.) Schr.; N.S. (1).

Puccinia veratri (DC.) Duby.

O-I On Chamaenerion latifolium (L.) Sweet; B.C. (1).

Epilobium hornemannii Reichenb.; B.C. (1).

II-III On Veratum californicum Durand; B.C. (1).

$V$. eschscholtzianum (R. \& S.) Rydb.; B.C. (1).

Puccinia vexans Farl.

II-III On Bouteloua gracilis (H.B.K.) Lag.; Man. (2).

Puccinia vincae (DC.) Berk.

O-I-II-III On Vinca major L.; Ont. (1).

Puccinia violae (Schum.) DC.

O-I-II-III On Viola adunca J. E. Sm.; Man. (2).

V. canadensis L.; Alta., B.C. (1), Man., Sask. (2), Que. (5).

$V$. conspersa Riechenb.; Que. (1).

$V$. eriocarpa Schw.; Ont. (1).

V. nephrophylla Greene; Alta. (1), Sask. (2), Que. (4).

V. odorata L.; Man. (2), B.C. (21). 
V. pedatifida G. Don.; Man., Sask. (2).

$V$. pubescens Ait.; Ont. (3).

V. renifolia Gray; Sask. (2).

$V$. septentrionalis Greene; N.S., Ont. (1).

$V$. sororia Willd.; Ont. (1).

V. tricolor L.; Man. (2), B.C. (23).

Viola spp., B.C.; Sask., Man., N.B., N.S. (2).

Puccinia virgaureae (DC.) Lib.

III On Solidago puberula Nutt.; N.S. (1).

Puccinia waldsteiniae Curt.

III On Waldsteinia fragarioides (Michx.) Tratt.; Ont. (1).

Puccinia windsoriae Schw.

II-III On Triodia flava (L.) Smyth; Ont. (1).

Puccinia xanthii Schw.

III On Ambrosia coronopifolia T. \& G.; Man. (2).

A. trifida L.; Ont. (1), Man. (2).

Xanthium commune Britt.; Man. (2).

$X$. chinense Mill.; Ont. (1).

Puccinia ziziae E. \& E.

III On Zizia cordata (Walt.) Koch; Sask. (2).

Pucciniastrum agrimoniae (Schw.) Tranz.

II-III On Agrimonia gryposepala Wallr.; N.S. (1), Alta., Man. (25), Que. (4).

A. pubescens Wallr.; Ont. (1).

A. striata Michx.; Alta. (1).

Agrimonia sp.; N.S. (31).

Pucciniastrum americanum (Farl.) Arth.

O-I On Picea glauca (Moench) Voss (P. canadensis (Mill.) B.S.P.); Ont. (1).

II-III On Rubus melanolasius Focke; B.C. (1).

R. strigosus Michx.; N.S., Ont., Que. (1).

Rubus spp.; P.E.I. (4), Que. (5), N.B. (23).

Pucciniastrum arcticum (Lagerh.) Tranz.

O-I On Picea glauca (Moench) Voss (P. canadensis (Mill.) B.S.P.); Alta., Ont. (1).

II-III On Rubus acaulis Michx.; Sask. (2).

$R$. arcticus L.; Man. (2).

$R$. pubescens Raf.; Man. (25).

R. iriflorus Richards.; Alta., Man., N.B., Ont. (1), Sask. (2).

Rubus spp.; Ont., P.E.I. (23).

Pucciniastrum galii (Link) E. Fisch.

II-III On Galium triflorum Michx.; B.C. (1).

Pucciniastrum goeppertianum (Kuehn) Kleb.

O-I On Abies balsamea (L.) Mill.; N.S., Que., Sask. (1).

A. lasiocarpa (Hook.) Nutt.; Alta., B.C. (1).

III On Vaccinium myrtillus L.; Alta. (1).

V. oreophilum Rydb.; Alta. (1).

$V$. pennsylvanicum Lam.; N.S., Nfld. (1).

V. vitis-idaea L.; Sask. (2).

Vaccinium spp.; Ont., Man. (2), Que., N.B., N.S. (23). 
Pucciniastrum myrtilli (Schum.) Arth.

O-I On Tsuga canadensis (L.) Carr.; N.S. (1).

II-III On Rhodora canadensis (L.) Torr.; N.S. (1).

Gaylussacia baccata (Wang.) K. Koch; Que. (1).

Vaccinium corymbosum L., Ont. (1).

V. myrtillus L.; Alta. (1).

V. pennsylvanicum Lam.; N.S. (1).

Pucciniastrum potentillae Kom.

II-III On Potentilla tridentata Ait. (Sibbaldiopsis t. (Ait.) Rydb.); Man. (2), N.B., Ont. (1).

Pucciniastrum pustulatum (Pers.) Diet.

O-I On Abies amabilis (Loud.) Forbes; B.C. (1).

A. balsamea (L.) Mill.; Alta., N.S. (1), Ont. (24).

A. lasiocarpa (Hook.) Nutt.; B.C. (1).

II-III On Chamaenerion spicatum (Lam.) S. F. Gray; Alta., B.C., Man., N.S., N.W.T., Sask., Nfld. (1).

Clarkia spp.; Sask., P.E.I. (23).

Epilobium adenocaulon Haussk.; Ont. (24), Man., Sask. (2), B.C. (1).

Epilobium spp.; Ont. (1), Que. (5).

Godetia spp.; Alta., Sask., Man., Ont., Que., P.E.I. (23).

Pucciniastrum pyrolae (Pers.) Schroet.

II-III On Moneses uniflora (L.) Gray; Alta. (1).

Pyrola asarifolia Michx.; Alta., Man. (1), Sask. (2).

P. chlorantha Sw.; Alta. (1), Man. (25).

P. elliptica Nutt.; Alta., N.S., Ont., Sask. (1).

$P$. rotundifolia Michx.; Man. (2).

$P$. secunda L.; Alta., Ont. (1).

Pucciniastrum sparsum (Wint.) E. Fisch.

II-III On Arctostaphylos alpina (L.) Spreng.; B.C. (1), Man. (2).

Arbutus menziesii Pursh; B.C. (6).

Tranzschelia fusca (Pers.) Diet.

O-III On Anemone quinquefolia L.; Ont. (1).

Tranzschelia pruni-spinosae (Pers.) Diet.

O-I On Anemone coronaria L.; B.C. (19).

A. quinquefolia L.; Ont., Que. (1).

Hepatica acutiloba DC.; Que. (1).

Ranunculus recurvatus Poir.; B.C. (1).

II-III On Padus nana (DuRoi) Roem., Ont. (1).

Prunus domestica L.; B.C. (23).

Tranzschelia suffusca (Holw.) Arth.

O-III On Anemone patens L., var. wolfgangiana (Besser) Koch; Man.,

Sask. (2).

Pulsatilla ludoviciana (Nutt.) Heller; Sask. (1).

Tranzschelia thalictri (Chev.) Diet.

O-III On Thalictrum dasycarpum Fisch. \& Lall.; Man. (2).

T. polygamum Muhl.; N.S., Ont. (1).

T. venulosum Trel.; Sask. (2).

Thalictrum sp.; Que. (Collected by A. Payette, July 1949.) 
Uraecium holwayi Arth.

O-I On Tsuga heterophylla (Raf.) Sarg.; B.C. (1).

T. mertensiana (Bong.) Carr.; B.C. (1).

Uredinopsis macrosperma (Cooke) Magn.

O-I On Abies sp.; B.C. (12).

II-III On Pteridium aquilinum (L.) Kuehn var. pubescens Underw.; B.C. (1).

Uredinopsis mirabilis (Peck) Magn.

O-I On Abies balsamea (L.) Mill.; N.S., Nfid., Ont. (1)

II-III On Onoclea sensibilis L.; N.S., Ont., Que. (1).

Uredinopsis osmundae Magn.

O-I On Abies balsamea (L.) Mill.; N.S. (1), Ont. (24).

II-III On Osmunda claytoniana L.; N.S. (1), Ont. (24).

O. spectabilis Willd.; N.S. (1).

Uredinopsis phegopteridis Arth.

O-I On Abies balsamea (L.) Mill.; N.S. (1), Ont. (24).

II-III On Phegopteris dryopteris (L.) Fée; Alta., N.S. (1), Ont. (24).

Uredinopsis struthiopteridis Stoermer

O-I On Abies balsamea (L.) Mill.; N.S. (1), Man., Sask. (2), Ont. (24).

II-III On Athyrium cyclosorum Rupr., N.S., Ont. (1)

Filix bulbifera (L.) Underw., Ont. (1).

Pteretis nodulosa (Michx.) Nieuwl.; Alta., N.S., Que., Nfid.

(1), Man. (2).

Dryopteris thelypteris (L.) Gray; N.S., Ont. (1).

Uromyces abbreviatus Arth.

O-III On Psoralea physodes Dougl.; B.C. (1).

Uromyces acuminatus Arth.

II-III On Spartina alterniflora Loisel., N.S. (1).

S. gracilis Trin.; Alta. (1), Sask. (2).

S. pectinata Link (S. michauxiana Hitchc.); Man., N.S. (1), Sask. (25).

U. acuminatus var. magnatus (Arth.) J. J. Davis

O-I On Maianthemum canadense Desf.; N.S. (1), Sask. (2).

Smilacina stellata (L.) Desf.; Sask. (2).

U. acuminatus var. polemonii J. J. Davis

O-I On Collomia linearis Nutt., Sask. (2).

Phlox sp., Sask. (25).

U. acuminatus var, spartinae (Farl.) Arth.

O-I On Moehringia lateriflora (L.) Fenzl; N.S. (1).

Spergularia canadensis (Pers.) D. Don; N.S. (1).

U. acuminatus var. steironematis (Arth.) J. J. Davis

O-I On Dodecatheon pauciflorum (Durand) Greene; Sask. (1).

Uromyces alopecuri Seym.

O-I On Ranunculus macounii Britt.; Man. (25), Sask. (2).

R. micranthus Nutt.; Alta. (1).

$R$. scleratus L.; Man. (25).

II-III On Alopecurus aristulatus Michx.; Man., Sask. (25).

A. geniculatus L. var. aristulatus Torr.; Man. (2). 
Uromyces amoenus Syd.

III On Anaphalis subalpina (Gray) Rydb.; B.C. (1).

Uromyces armeriae (Schlecht.) Lév.

U. armeriae ssp. hudsonicus Savile \& Conners

O-I-II-III On Armeria maritima Willd. var. labradorica Wallr.,; Que. (30).

U. armeriae ssp. pacificus Savile \& Conners

I-II-III On Armeria maritima Willd.; B.C. (30).

Uromyces asclepiadis (Schw.) Cooke

II-III On Asclepias incarnata L.; Ont. (1).

A. syriaca L.; Ont. (1).

Uromyces betae (Pers.) Lév.

II-III On Beta vulgaris L.; B.C. (12).

Uromyces caladii (Schw.) Farl.

O-I-II-III On Arisaema triphyllum (L.) Schott; N.S., Ont., Que. (1). Muricauda dracontium (L.) Small; Ont. (1).

Uromyces caryophyllinus (Schr.) Wint.

II-III On Dianthus caryophyllus L.; Man. (2), Sask. (25), all provinces (23).

Uromyces coloradensis Ell. \& Ev.

$U$. coloradensis var. campester Arth.

O-I-III On Vicia americana Muhl., Alta. (1), Man. (2).

V. cracca L.; Man. (2).

$V$. oregana Nutt.; Ont. (1).

$V$. sparsifolia Nutt.; Sask. (2).

$U$. coloradensis var. maritimus Arth.

O-I-III On Vicia americana Muhl.; B.C. (1).

Uromyces dactylidis Otth

O-I On Ranunculus repens L.; N.S. (1).

II-III On Dactylis glomerata L.; N.S. (23).

Poa crocata Michx.; Alta. (1).

P. pratensis L.; Alta. (1).

P. trivialis L.; N.S. (1).

Uromyces fabae (Pers.) de Bary

O-I-II-III On Lathyrus myrtifolius Muhl; Ont. (1).

L. ochroleucus Hook.; Alta., Ont. (1), Man., Sask. (25).

L. venosus Muhl.; Alta. (1), Sask. (25), Man. (2).

Pisum sativum L.; Man., Sask. (25), B.C., P.E.I., Que. (12),

Ont., Que., N.B., N.S. (23).

Vicia americana Muhl.; Man., Alta. (25), B.C. (1).

$V$. angustifolia Reichard; B.C. (1).

V. cracca L.; N.S., Que. (1).

$V$. oregana Nutt.; Alta. (25).

V. sativa L.; B.C., Alta. (23).

V. sparsifolia Nutt.; Alta. (1).

Uromyces gentianae Arth.

II-III On Gentiana strictiflora (Rydb.) Nels.; Sask. (2).

Pleurogyne rotata (L.) Griesb.; Que. (1).

Uromyces geranii (DC.) Fries

O-I-II-III On Geranium spp.; P.E.I. (12), Ont. (23). 
Uromyces glycyrrhizae (Rab.) Magn.

O-I-III On Glycyrrhiza lepidota Nutt.; Alta. (1), Man., Sask. (25).

Uromyces hedysari-obscuri (DC.) Car. \& Picc.

O-I-II-III On Hedysarum americanum (Michx.) Britt.; Alta. (1).

H. boreale Nutt.; Alta. (1), Man., Sask. (2).

H. cinerascens Rydb.; Alta. (1), Sask. (2).

H. mackenzii Richards.; Alta. (1).

H. sulphurascens Rydb.; Alta. (1).

Uromyces hedysari-paniculati (Schw.) Farl.

O-I-II-III On Desmodium dillenii Darl.; Ont. (1).

Uromyces heterodermus Syd.

O-III On Erythronuim grandiflorum Pursh; B.C. (1).

Uromyces holwayi Lagerh.

O-I-II-III On Lilium canadense L.; Ont. (1).

L. columbianum Hanson; B.C. (1).

L. michiganense Farwell; Ont. (1).

Uromyces houstoniatus (Schw.) Sheldon

O-I On Houstonia longifolia Gaertn.; Sask. (1).

Uromyces hyperici (Spreng.) Curt.

O-I-II-III On Hypericum kalmianum L.; Ont. (1).

H. perforatum L.; Man. (2).

H. prolificum L.; Ont. (1).

H. virginicum L.; (Triadenum v. (L.) Raf.); N.S., Ont., Que. (1).

Uromyces intricatus Cooke

O-I-III On Eriogonum flavum Nutt.; Sask. (2).

Uromyces junci (Desm.) L. Tul.

O-I On Aster spp.; Sask., Ont. (1).

Helianthus petiolaris Nutt.; Man., Sask. (25).

H. subrhomboideus Rydb.; Sask. (2).

H. subtuberosus Bourg.; Alta. (1).

H. tuberosus L.; Man. (1).

Cirsium flodmanii (Rydb.) Arth.; Man. (1), Sask. (2).

C. megacephalum (Gray) Cockerell; Sask. (2).

C. undulatum (Nutt.) Spreng.; Alta. (1), Sask. (25).

Arnica chamissonis Less.; Alta. (1).

II-III On Juncus ater Rydb.; Alta. (1), Sask. (25).

J. balticus Willd.; Man., Sask. (25).

J. dudleyi Wieg.; Man. (25).

J. filiformis L.; Sask. (25).

Uromyces junci-effusi Syd.

II-III On Juncus filiformis L.; N.S. (1).

J. xiphioides E. Mey.; B.C. (1).

Uromyces lapponicus Lagerh.

O-I-III On Astragalus macounii Rydb.; Alta. (1).

Astragalus sp.; Alta. (1).

Phaca frigida L.; N.W.T. (1).

Uromyces lespedezae-procumbentis (Schw.) Curt.

O-I-II-III On Lespedeza capitata Michx.; Ont. (1). 
Uromyces limonii (DC.) Lév.

O-I-II-III On Limonium latifolium; B.C. (30).

L. vulgare; Ont. (30).

Uromyces limonii-caroliniani Savile \& Conners

O-I-II-III On Limonium nashii Small (not L. carolinianum (Walt.) Britt.); Que., N.B., N.S. (30).

Uromyces lupini Berk. \& Curt.

O-I-II-III On Lupinus sp.; B.C. (13).

Uromyces minor Schroet.

I-III On Trifolium dubium Sibth.; B.C. (18).

T. microdon H. \& A.; B.C. (1).

T. oliganthum Steud.; B.C. (1).

Uromyces miurae Syd.

III On Fritillaria kamtschatcensis Ker.; B.C. (1).

Uromyces nerviphilus (Grognot) Hotson

O-I-III On Trifolium hybridum L.; Sask. (2).

T. pratense L.; Ont. (23).

T. repens L.; Ont. (1). Man. (2).

Uromyces peckianus Farl.

O-I On Atriplex patula L.; N.S. (1).

Chenopodium album L.; N.S. (1).

Salicornia herbacea L.; N.S. (1).

S. pacifica Standl.; B.C. (1).

Suaeda maritima (L.) Dumort; N.S. (1).

Plantago eriopoda Torr.; Alta. (1).

II-III On Distichlis spicata (L.) Greene; B.C., N.S. (1).

Uromyces perigynius Halst.

II-III On Rudbeckia ampla A. Nels.; Man. (6).

O-I On R. laciniata L.; Man. (1).

Uromyces phaseoli (Pers.) Wint.

O-I-II-III On Phaseolus vulgaris L.; Ont. (1), B.C. (13), P.E.I. (12), N.B. (19), Que., N.S. (23).

Uromyces plumbarius Peck

O-I-II-III On Gaura coccinea Nutt.; Alta. (1), Sask. (25), Man. (2).

Uromyces polygoni (Pers.) Fckl.

O-I-II-III On Polygonum aviculare L.; Ont., Que. (1), Alta. (6), P.E.I. (4), Man. (2).

$P$. buxiforme Small; Sask. (2).

P. erectum L.; Man. (2).

P. fowleri Rob.; Nfld. (1).

P. ramosissimum Michx.; Man. (2).

P. rubescens Small; Sask. (2).

P. spergulariaeforme Meissn.; B.C. (1).

Uromyces proëminens (DC.) Pass.

$U$. proëminens var. euphorbiicola (Tranz.) Arth.

O-I-II-III On Chamaesyce humistrata (Engelm.) Small; Ont. (1). 
U. proeminens var. proëminens

O-I-II-III On Chamaesyce glyptosperma (Engelm.) Small; Sask. (25).

C. hirsuta (Torr.) Arth.; Ont. (1).

C. serphyllifolia (Pers.) Small; B.C. (1), Man. (25).

Uromyces psoraleae Peck

U. psoraleae var. argophyllae (Seym.) Arth.

O-I-III On Psoralea argophylla Pursh; Sask. (2).

$U$. psoraleae var. psoraleae

O-I-III On Psoralea lanceolata Pursh; Sask. (2).

Uromyces punctatus Schroet.

II-III On Oxytropis gracilis (A. Nels.) K. Schum.; Man. (2).

Uromyces pyriformis Cooke

(See note under $U$. sparganii)

II-III On Acorus calamus L.; Ont. (1).

Uromyces rhyncosporae Ellis

II-III On Rhynchospora alba (L.) Vahl; Ont. (1).

Uromyces rudbeckiae Arth. \& Bisby

III On Rudbeckia laciniata L.; Man. (2), Ont. (1).

Uromyces scirpi (Cast.) Burr.

O-I On Cicuta occidentalis Greene; Sask. (2).

Sium cicutaefolium Schrank; Man., Sask. (2).

II-III On Scirpus fluviatilis (Torr.) Gray; Que. (1).

S. paludosus Nels.; N.S. (1), Sask. (2).

S. validus Vahl; N.S., Ont. (1).

(According to G.B. Cummins (Mycologia 27:611. 1935) the rust on Scirpus validus is $U$. americanus Speg., not $U$. scirpi).

Uromyces silphii (Burr.) Arth. -

II-III On Juncus longistylis Torr.; Man. (1), Sask. (2).

J. macer S. F. Gray; Sask. (2), Ont. (1).

Uromyces sparganii Cooke \& Peck

II-III On Sparganium eurycarpum Englem.; Ont., Que. (1).

(J. A. Parmelee and D. B. O. Savile (Mycologia 46: 823-826. 1954) report that $U$. pyriformis on Acorus calamus is indistinguishable from $U$. sparganii ssp. sparganii and have demonstrated that the O-I states, which were found in Ont. and Que., occur on Hypericum virginicum. The II-III states were also reported on A. calamus in Que., N.S., and on S. eurycarpum in N.S.).

Uromyces spragueae Hark.

I-III On Lewisia rediviva Pursh; B.C. (5).

(D. B. O. Savile and J. A. Parmelee (Mycologia 1956, in press) have placed the rust on $L$. rediviva in B.C. in $U$. unitus Peck ssp. unitus).

Uromyces striatus Schroet.

O-I On Euphorbia cyparissias L.; Ont. (18).

II-III On Medicago lupulina L.; Ont. (1).

M. sativa L.; Man. (2), Sask. (18), Ont., N.S., P.E.I. (23). 
Uromyces trifolii (Hedw. f.) Lév.

U. trifolii var. trifolii-repentis (Liro) Arth.

O-I-II-III On Trifolium repens L.; Nfld., Man. (1), Alta. (2), P.E.I. (12), B.C., Sask., Ont., Que. (23).

T. incarnatum L.; P.E.I. (23).

U. trifolii var. hybridi (W. H. Davis) Arth.

O-I-II-III On Trifolium hybridum L.; Ont. (1), Man., Sask., Alta. (2), All provinces (23).

U. trifolii var. fallens (Desm.) Arth.

O-I-II-III On Trifolium medium L.; Man. (2).

T. pratense L.; Man., B.C., Ont., Que., N.S. (1), N.B., P.E.I. (23).

Uromyces zygadeni Arth.

O-I-II-III On Zygadenus gramineus Rydb.; Alta. (1).

Uropyxis amorphae (Curt.) Schroet.

II-III On Amorpha canescens Pursh.; Man. (2).

A. fruticosa L.; Man. (25).

A. nana Nutt.; Man. (2).

Uropyxis petalostemonis (Farl.) de Toni

O-I-II-III On Petalostemon oligophyllus (Torr.) Rydb.; Sask. (2). 


\section{A LIST OF HOSTS AND THE RUSTS THAT MAY BE EXPECTED TO OCCUR ON THEM}

Abies amabilis (Loud.) Forbes

Pucciniastrum pustulatum

Abies balsamea (L.) Mill.

Hyalopsora aspidiotus

Melampsora abieti-capraearum

Melampsorella cerastii

Milesia fructuosa

M. marginalis

M. polypodophila

Pucciniastrum goeppertianum

P. pustulatum

Uredinopsis macrosperma

U. mirabilis

U. osmundae

U. phegopteridis

U. struthiopteridis

Abies grandis Lindl.

Melampsorella cerastii

Abies lasiocarpa (Hook.) Nutt.

Melampsorella cerastii

Pucciniastrum pustulatum

P. goeppertianum

Abies sp.

Uredinopsis macrosperma

Achillea millefolium L.

Puccinia millefolii

Achillea multiflora Hook.

Puccinia millefolii

Achillea ptarmica L.

Pucccinia millefolii

Acorus calamus L.

Uromyces pyriformis

Actaea alba (L.) Mill.

Puccinia rubigo-vera var. agropyri

Actaea brachypoda Ell.

Puccinia rubigo-vera var. agropyrina

Actaea rubra (Ait.) Willd.

Puccinia rubigo-vera var. agropyri

Adoxa moschatellina L.

Puccinia argentata

Adoxa sp.

Puccinia adoxae

Aegilops cylindrica Host, cult. Puccinia glumarum

Aegilops sp., cult.

Puccinia glumarum

P. rubigo-vera var. tritici

Agoseris glauca (Pursh) Raf.

Puccinia extensicola var. hieraciata

P. hieracii

P. maculosa

Agrimonia gryposepala Wallr. Pucciniastrum agrimoniae

Agrimonia pubescens Wallr.

Pucciniastrum agrimoniae
Agrimonia striata Michx.

Pucciniastrum agrimoniae

Agrimonia sp.

Pucciniastrum agrimoniae

Agropyron albicans Scribn. \& Sm.

Puccinia montanensis

P. rubigo-vera var. agropyrina

Agropyron cristatum (L.) Gaertn., cult.

Puccinia glumarum

P. graminis

Agropyron dasystachyum (Hook.) Scribn.

Puccinia glumarum

P. graminis

P. montanensis

P. rubigo-vera var. agropyri

Agropyron desertorum Schult.

Puccinia glumarum

Agropyron elongatum Host

Puccinia glumarum

Agropyron griffithsii Scribn. \& Sm.

Puccinia glumarum

P. graminis

Agropyron obtusiusculum Lange

Puccinia glumarum

Agropyron repens (L.) Beauv.

Puccinia coronata

P. glumarum.

P. graminis

P. montanensis

P. rubigo-vera var. agropyrina

Agropyron richardsonii (Trin.) Schrad.

Puccinia graminis

P. glumarum

P. montanensis

P. rubigo-vera var. agropyrina

Agropyron smithii Rydb.

Puccinia coronata

P. graminis

P. glumarum

P. montanensis

P. rubigo-vera var. agropyri

Agropyron spicatum (Pursh) Scribn. \& Sm.

Puccinia glumarum

P. rubigo-vera var. agropyri

Agropyron trachycaulum (Lk.) Malte (A. tenerum Vasey)

Puccinia coronata

P. glumarum

P. graminis

P. montanensis

P. rubigo-vera var. agropyri

P. rubigo-vera var. agropyrina

Agropyron violaceum (Hornem.) Lange

Puccinia rubigo-vera var. agropyri

Agrostis exarata 'Trin.

Puccinia ?praegracilis 
Agrostis hyemalis (Walt.) B.S.P.

Puccinia graminis

$P$. liatridis

Agrostis palustris Huds.

Puccinia graminis

Agrostis perennans (Walt.) Tuckerm.

Puccinia graminis

P. rubigo-vera var. impatientis

Agrostis stolonifera L.

Puccinia graminis

Agrostis tenuis Sibth.

Puccinia graminis

Agrostis thurberiana Hitchc.

Puccinia praegracilis var. praegracilis

Allium cernuum Roth

Puccinia granulispora

Allium textile Nels. \& Macbr.

Puccinia blasdalei

Allium sp.

Puccinia granulispora

P. porri

Alopecurus aristulatus Michx.

Uromyces alopecuri

Alopecurus geniculatus L. var. aristulatus Torr.

Uromyces alopecuri

Alopecurus pratensis L.

Puccinia graminis

P. rubigo-vera var. agropyrina

Althaea ficifolia (L.) Cav.

Puccinia malvacearum

Althae rosea (L.) Cav.

Puccinia malvacearum

Ambrosia psilostachya DC. (A. coronopifolia T. \& G.)

Puccinia xanthii

Ambrosia trifida L.

Puccinia xanthii

Amelanchier alnifolia Nutt.

Gymnosporangium clavariaeforme

G. clavipes

G. corniculans

G. juvenescens

G. nelsoni

Amelanchier canadensis (L.) Medic.

Gymnosporangium clavariaeforme

Amelanchier intermedia Spach

Gymnosporangium clavipes

G. nidus-avis

Amelanchier florida Lindl.

Gymnosporangium juvenescens

Amelanchier oligocarpa (Michx.) Roem.

Gymnosporangium clavipes

Amelanchier pumila Nutt.

Gymnosporangium juvenescens

Amelanchier sanguinea (Pursh) DC.

Gymnosporangium corniculans

G. clavipes

Amelanchier spicata

Gymnosporangium clavariaeforme

Amelanchier spp.

Gymnosporangium clavipes
Amorpha canescens Pursh

Uropyxis amorphae

Amorpha fruticosa L.

Uropyxis amorphae

Amorpha nana Nutt. Uropyxis amorphae

Anaphalis subalpina (Gray) Rydb. Uromyces amoenus

Andropogon provincialis Lam.

Puccinia andropogonis var. pustulata P. ellisiana

Andropogon scoparius Michx.

Puccinia andropogonis var. pustulata

P. ellisiana

Anemone canadensis L.

Puccinia anomones-virginianae

P. magnusiana

Anemone coronaria L.

Tranzschelia pruni-spinosae

Anemone cylindrica Gray

Puccinia gigantispora

P. rubigo-vera var. agropyri

$\mathrm{P}$. rubigo-vera var. agropyrina

Anemone globosa Nutt.

Puccinia gigantispora

P. rubigo-vera var. agropyri

P. rubigo-vera var. agropyrina

Anemone husdoniana Richards.

Puccinia rubigo-vera var. agropyrina

Anemone parviflora Michx.

Puccinia pulsatillae

Anemone patens L. var. wolfgangiana (Besser) Koch

Puccinia pulsatillae

Tranzschelia suffusca

Anemone quinquefolia L.

Puccinia rubigo-vera var. agropyrina

Tranzschelia fusca

T. pruni-spinosae

Anemone riparia Fern.

Puccinia anemones-virginianae

P. rubigo-vera var. agropyrina

Anemone virginiana $L$.

Puccinia anemones-virginianae

$P$. rubigo-vera var. agropyri

Anthoxanthum odoratum L.

Puccinia poae-nemoralis

Antirrhinum majus L., cult.

Puccinia antirrhini

Apargia autumnalis (L.) Hoffm.

Puccinia hieracii

Aquilegia flavescens Wats.

Puccinia rubigo-vera var. agropyri

Aquilegia formosa Fisch.

Puccinia rubigo-vera var. agropyri

Arabis brachycarpa (Torr. \& Gray) Britt.

Puccinia monoica

Arabis divaricarpa Nels.

Puccinia holboellii

Arabis drummondii Gray

Puccinia holboellii

P. monoica 
Arabis lignipes Nels.

Puccinia holboellii

Arabis lyallii Wats.

Puccinia holboellii

Arabis lyrata L.

Puccinia monoica

Arabis nuttallii B. L. Rob.

Puccinia monoica

Arabis ovata (Pursch) Poir.

Puccinia monoica

Arabis retrof racta Grah.

Puccinia monoica

Arabis sp.

Puccinia monoica

Aralia nudicaulis L.

Nyssopsora clavellosa

Arbutus menziesii Pursh

Pucciniastrum sparsum

Arctium minus Schk.

Puccinia bardanae

Arctostaphylos alpina (L.) Spreng.

Pucciniastrum sparsum

Arctostaphylos uva-ursi (L.) Spreng.

Chrysomyxa arctostaphyli

Arisaema triphyllum (L.) Schott.

Uromyces caladii

Armeria maritima Willd., cult.

Uromyces armeriae ssp. pacificus

Armeria maritima Willd. var. labradorica Wallr.

Uromyces armeriae ssp. hudsonicus

Arnica chamissonis Less.

Uromyces junci

Aronia arbutifolia (L.) Ell.

Gymnosporangium clavipes

Aronia melanocarpa (Michx.) Ell. Gymnosporangium clavipes

Artemisia biennis Willd.

Puccinia atrof usca

Artemisia camporum Rydb.

Puccinia atrofusca

Artemisia cana Pursh

Puccinia absinthii

Artemisia caudata Michx.

Puccinia atrofusca

Artemisia frigida Willd.

Puccinia absinthii

P. atrof usca

P. millefolii

Artemisia glauca Pallas

Puccinia atrofusca

Artemisia gnaphalodes Nutt.

Puccinia absinthii

$P$. atrof usca

Artemisia ludoviciana Nutt.

Puccinia absinthii

Artemisia purshiana Besser

Puccinia atrofusca

Asarum caudatum Lindl.

Puccinia asarina

Asclepias incarnata L.

Uromyces asclepiadis
Asclepias syriaca $L$.

Uromyces asclepiadis

Asparagus officinalis L., cult.

Puccinia asparagi

Aster conspicuus Lindl.

Coleosporium solidaginis

Aster cordifolius L.

Coleosporium solidaginis

Puccinia asteris

Aster ericoides L.

Coleosporium solidaginis

Puccinia asteris

Aster junceus Ait.

Coleosporium solidaginis

Aster laevis L.

Coleosporium solidaginis

Puccinia asteris

Aster lateriflorus (L.) Britt.

Coleosporium solidaginis

Aster lindleyanus Torr. \& Gray

Coleosporium solidaginis

Puccinia asteris

Aster novae-angliae L.

Coleosporium solidaginis

Puccinia asteris

Aster occidentalis Nutt.

Coleosporium solidaginis

Aster paniculatus Lam.

Coleosporium solidaginis

Aster salicifolius Lam.

Coleosporium solidaginis

Aster umbellatus Mill.

Coleosporium solidaginis

Aster spp.

Puccinia extensicola var. asteris

Uromyces junci

Astragalus macounii Rydb. Uromyces lapponicus

Astralagus sp.

Uromyces lapponicus

Athyrium cyclosorum Rupr.

Uredinopsis struthiopteridis

Atriplex patula L.

Uromyces peckianus

Avena fatua $L$.

Puccinia coronata

$P$. graminis

Avena nuda L.

Puccinia graminis

Avena sativa L., cult.

Puccinia coronata

P. graminis

Balsamorrhiza sagittata (Pursh) Nutt. Puccinia balsamorhizae

Beckmannia erucaeformis (L.) Host. Puccinia coronata

Beckmannia syzigachne (Steud.) Fern.

Puccinia coronata

P. graminis

Berberis aquifolium Pursh (Mahonia a. (Pursh) Nutt.), cult.

Puccinia graminis 
Berberis vulgaris L., cult. and escaped Puccinia graminis

Berberis spp.

Puccinia graminis

Beta vulgaris $L$.

Uromyces betae

Betula populifolia Marsh.

Melampsoridium betulinum

Betula pumila L.

Melampsoridium betulinum

Betula sp.

Melampsoridium betulinum

Bistorta vivipara (L.) S. F. Gray

Puccinia bistortae

$P$. septentrionalis

Bouteloua gracilis (H.B.K.) Lag.

Puccinia vexans

Briza maxima L.

Puccinia graminis

Briza minor L.

Puccinia graminis

Bromus carinatus Thurb.

Puccinia glumarum

Bromus carinatus hookerianus (Thurb.) Shear Puccinia rubigo-vera var. agropyri

Bromus ciliatus L.

Puccinia coronata

P. glumarum

P. rubigo-vera var. agropyri

P. rubigo-vera var. agropyrina

Bromus ciliatus laeviglumis Scribn.

Puccinia rubigo-vera var. agropyrina

Bromus hordeaceus L.

Puccinia graminis

Bromus latiglumis (Shear) Hitchc.

Puccinia coronata

$P$. rubigo-vera var. agropyrina

Bromus marginatus Nees.

Puccinia glumarum

Bromus porteri (Coulter) Nash

Puccinia coronata

P. rubigo-vera var. agropyri

Bromus pumpellianus Scribn.

Puccinia coronata

P. graminis

P. rubigo-vera var. agropyri

Bromus purgans L.

Puccinia rubigo-vera var. agropyrina

Bromus sitchensis Trin., cult.

Puccinia glumarum

Bromus spp.

Puccinia glumarum

Calamagrostis canadensis (Michx.) Beauv. Puccinia coronata

Calamagrostis elongata (Kearney) Rydb. Puccinia coronata

Calamagrostis montanensis Scribn. Puccinia coronata

Calamagrostis rubescens Buckl.

Puccinia coronata
Calamovilfa longifolia (Hook.) Hack. Puccinia amphigena

Callistephus chinensis Nees, cult. Coleosporium solidaginis

Caltha leptosepala DC. Puccinia areolata

P. treleasiana

Caltha palustris $\mathrm{L}$.

Puccinia calthae

P. calthaecola

Campanula persicifolia L. Coleosporium campanulae

Campanula rapunculoides $\mathrm{L}$.

Coleosporium campanulae

Campanula rotundifolia L., var. intercedens Witasek

Puccinia campanulae

Campanula spp.

Coleosporium campanulae

Cardamine bellidifolia L.

Puccinia cruciferarum

Cardamine douglasii (Torr.) Britt.

Puccinia arabicola

Carex aquatilis Wahl.

Puccinia caricis-shepherdiae

Carex atherodes Spreng.

Puccinia caricis-shepherdiae

Carex douglasii Boott

Puccinia atrofusca

Carex eburnea Boott

Puccinia caricis-shepherdiae

Carex eleocharis Bailey

Puccinia atrofusca

Carex filifolia Nutt.

Puccinia atrofusca

Carex intumescens Rudge

Puccinia bolleyana

Carex lanuginosa Michx.

Puccinia caricis-shepherdiae

Carex lasiocarpa Ehrh.

Puccinia minutissima

Carex limosa L.

Puccinia limosae

Carex lupuliformis Sartw.

Puccinia bolleyana

Carex paupercula Michx.

Puccinia karelica

Carex praegracilis Boott

Puccinia atrofusca

Carex rossii Boott

Pucinia atrof usca

Carex sartwellii Dewey

Puccinia extensicola var. oenotherae

Carex scoparia Schk.

Puccinia extensicola var. euthamii

Carex sprengelii Dewey

Puccinia extensicola var. hieraciata

P. extensicola var, phrymae

Carex stratiformis Britt.

Puccinia limosae

Carex substricta (Kuek.) Mack.

Puccinia caricis-shepherdiae

P. minutissima 
Carex vesicaria $\mathrm{L}$.

Puccinia caricus var. urticata

P. caricis-shepherdiae

Carex spp.

Puccinia caricis var. grossulariata

P. caricis var. uniporula

P. caricis var. urticata

P. extensicola var. asteris

P. extensicola var. hieraciata

P. extensicola var. solidaginis

$P$. extensicola var. erigerontis

Carthamus tinctorius L.

Puccinia carthami

Castilleja sessiliflora Pursh

Puccinia andropogonis var. micropuncta

Centaurea cyanus L.

Puccinia cyani

Cephalanthus occidentalis $\mathrm{L}$.

Puccinia seymouriana

Cerastium alpinum L.

Melampsorella cerastii

Cerastium arvense L.

Melampsorella cerastii

Cerastium berringianum C. \& S.

Melampsorella cerastii

Cerastium vulgatum $\mathrm{L}$.

Melampsorella cerastii

Chamaedaphne calyculata (L.) Moench

Chrysomyxa cassandrae

C. ledi var. cassandrae

Chamaenerion latifolium (L.) Sweet Puccinia veratri

Chamaenerion spicatum (Lam.) S. F. Gray

Pucciniastrum pustulatum

Puccinia extensicola var. oenotherae

$P$. gigantea

Chamaesyce glyptosperma (Engelm.) Small Uromyces proëminens var. proëminens

Chamaesyce hirsuta (Torr.) Arth.

Uromyces proëminens var. proëminens

Chamaesyce humistrata (Englem.) Snall

Uromyces proëminens var. euphorbiicola

Chamaesyce serphyllifolia (Pers.) Small

Uromyces proëminens var. proëminens

Chenopodium album $\mathrm{L}$.

Puccinia aristidae

Uromyces peckianus

Cheirinia cheiranthoides (L.) Link Puccinia aristidae

Chiogenes hispidula (L.) T. \& G. Chrysomyxa chiogenis

Chrysopsis hirsutissima Greene Puccinia stipae

Cichorium intybus L.

Puccinia hieracii

Cicuta maculata L. Puccinia cicutae

Cicuta occidentalis Greene

Puccinia cicutae

Uromyces scirpi

Cinna arundinacea L.

Puccinia graminis
Cinna latifolia (Trev.) Gries.

Puccinia graminis

Circaea alpina L.

Puccinia circaeae

Circaea latifolia Mill.

Puccinia circaeae

Circaee pacifica Asch. \& Magn.

Puccinia circaeae

Cirsium arvense (L.) Scop. Puccinia suaveolens (P. obtegens)

Cirsium flodmanii (Rydb.) Arth. Puccinia cirsii

Uromyces junci

Cirsium lanceolatum (L.) Hill Puccini cnici

Cirsium megacephalum (Gray) Cockerell Uromyces junci

Cirsium undulatum (Nutt.) Spreng. Puccinia cirsii Uromyces junci

Cirsium spp. Puccinia cirsii

Clarkia spp. Pucciniastrum pustulatum

Claytonia caroliniana Michx. Puccinia mariae-wilsoni

Claytonia virginica $L$. Puccinia mariae-wilsoni

Clematis ligusticifolia Nutt. Puccinia rubigo-vera var. agropyri

Clinopodium vulgare $\mathrm{L}$. Puccinia menthae

Clintonia borealis (Ait.) Raf. Puccinia mesomajalis

Clintonia uniflora (Schult.) Kunth Puccinia mesomajalis

Collomia linearis Nutt. Uromyces acuminatus var. polemonii

Comandra livida Richards. Cronartium comandrae Puccinia comandrae

Comandra pallida A. DC. Cronartium comandrae Puccinia andropogonis var. pustulata P. comandrae

Comandra umbellata (L.) Nutt. Cronartium comandrae Puccinia andropogonis var. pustulata

Convallaria sp. Puccinia sessilis

Convolvulus sepium L. Puccinia convolvuli

Cornus canadensis L. Puccinia porphyrogenita

Cotoneaster lucida Gymnosporangium clavipes

Crataegus chrysocarpa Ashe Gymnosporangium betheli G. clavariaeforme

Crataegus coccinea L. Gymnosporangium globosum

Crataegus douglasii Lindl. Gymnosporangium betheli 
Crataegus punctata Jacq.

Gymnosporangium clavipes

G. globosum

Crataegus tomentosa L.

Gymnosporangium globosum

Crataegus spp.

Gymnosporangium clavariaeforme

G. clavipes

G. globosum

G. tubulatum

G. betheli

Crepis runcinata (James) Torr. \& Gray

Puccinia hieracii

Cryptogramma acrostichoides R. Br. Milesia darkeri

Cryptotaenia canadensis (L.) DC.

Puccinia cryptotaeniae

P. microica

Cydonia olbonga Mill.

Gymnosporangium clavariaeforme

G. clavipes

Cydonia sp.

Gymnosporangium clavipes

Cyperus strigosus L.

Puccinia canaliculata

Cypripedium parviflorum Salisb.

Puccinia cypripedii

Dactylis glomorata L.

Puccinia graminis

Uromyces dactylidis

Dasiphora fruticosa (L.) Rydb. (Potentilla f. L.)

Phragmidium andersoni

Decodon verticillatus (L.) Ell.

Puccinia minutissima

Deschampsia atropurpurea (Wahl.) Scheele

$\mathrm{P}$. praegracilis var. connersii

Deschampia caespitosa (L.) Beauv.

Puccinia coronata

$P$. graminis

P. poae-sudeticae var. airae

Desmodium dillenii Darl.

Uromyces hedysari-paniculati

Dianthus caryophyllus L., cult.

Uromyces caryophyllinus

Distichlis spicata (L.) Greene

Puccinia aristidae

Uromyces peckianus

Dodecatheon jeffreyi Moore

Puccinia ortonii

Dodecatheon pauciflorum (Durand) Greene Puccinia ortonii

Uromyces acuminatus var. steironematis

Draba arabisans Michx.

Puccinia drabae

Draba incerta Payson

Puccinia drabae

Draba lutea Gilib.

Puccinia aristidae

Draba sp.

Puccinia drabae
Dryopteris marginalis (L.) Gray

Milesia marginalis

Dryopteris spinulosa (O. F. Muell.) Watt.

Milesia fructuosa

Dryopteris thelypteris (L.) Gray

Uredinopsis struthiopteridis

Elaeagnus angustifolia L.

Puccinia caricis-shepherdiae

Elaeagnus commutata Bernh.

Puccinia caricis-shepherdiae

P. coronata

Eleocharis palustris (L.) R. \& S. Puccinia eleocharidis

Eleocharis spp.

Puccinia eleocharidis

Elymus canadensis L.

Puccinia coronata

$P$. graminis

P. montanensis

$\mathrm{P}$. rubigo-vera var. impatientis

Elymus curvatus Piper

Puccinia graminis

P. montanensis

$P$. rubigo-vera var. agropyrina

Elymus dahuricus Turcz.

Puccinia graminis

P. montanensis

Elymus diversiglumis Scribn. \& Ball

Puccinia rubigo-vera var. agropyri

$P$. rubigo-vera var. agropyrina

Elymus glaucus Buckl.

Puccinia glumarum

Elymus howellii Scribn. \& Merr.

Puccinia glumarum

Elymus innovatus Beal

Puccinia coronata

Elymus jejunus (Ramaley) Rydb.

Puccinia montanensis

$P$. rubigo-vera var. agropyrina

Elymus macounii Vasey

Puccinia graminis

P. rubigo-vera var. agropyri

P. rubigo-vera var. agropyrina

Elymus marginalis Rydb.

Puccinia glumarum

Elymus vancouverensis Vasey

Puccinia rubigo-vera var. agropyri

Elymus virginicus L.

Puccinia graminis

P. rubigo-vera var. agropyrina

P. rubigo-vera var. impatientis

Empetrum atropurpureum Fern. \& Wieg. Chrysomyxa empetri

Empetrum eamesii Fern. \& Wieg. Chrysomyxa empetri

Empetrum nigrum L.

Chrysomyxa empetri

Epilobium adenocaulon Haussk.

Puccinia vagans var. epilobii-tetragoni Pucciniastrum pustulatum

Epilobium alpinum L. Puccinia epilobii 
Epilobium angustifolium L.

(Chamaenerion spicatum (Lam.) S.F. Gray)

Puccinia extensicola var. oenotherae

Pucciniastrum pustulatum

Epilobium coloratum Muhl.

Pucciniastrum pustulatum

Epilobium hirsutum L.

Pucciniastrum pustulatum

Epilobium hornemannii Reichenb.

Puccinia veratri

Epilobium minutum Lindl.

Puccinia oenotherae

Epilobium spp.

Puccinia epilobii

P. scandica

Pucciniastrum pustulatum

Puccinia vagans var. epilobii-tetragoni

Erigenia bulbosa (Michx.) Nutt.

Puccinia erigeniae

Eriogonum flavum Nutt.

Uromyces intricatus

Eriophorum angustifolium Roth

Puccinia angustata

Eriophorum viridicarinatum (Engelm.) Fern.

Puccinia angustata

Erysimum cheiranthoides L. (Cheirinia c. (L.) T. \& G.)

Puccinia aristidae

Erythronium grandiflorum Pursh

Uromyces heterodermus

Eupatorium perfoliatum L.

Puccinia eleocharidis

Eupatorium purpureum L.

Puccinia eleocharidis

Eupatorium purpureum var. maculatum (L.) Darl.

Puccinia eleocharidis

Euphorbia cyparissias L.

Uromyces striatus

Euthamia graminifolia (L.) Nutt. (Solidago g. (L.) Salish.)

Puccinia extensicola var. euthamii

Festuca elatior L.

Puccinia graminis

Festuca myuros L.

Puccinia graminis

Festuca ovina L.

Puccinia crandallii

Festuca rubra L.

Puccinia rubigo-vera var. agropyri

Festuca saximontana Rydb.

Puccinia crandallii

Festuca scabrella Torr.

Puccinia cockerelliana

Festuca spp.

Puccinia coronata

Filix bulbifera (L.) Underw.

Uredinopsis struthiopteridis

Filix fragilis (L.) Gilib.

Hyalopsora polypodii
Fluminia festucacea (Willd.) Hitchc.

(Scolochloa festucacea (Willd.) Link)

Puccinia coronata

Fraxinus americana L.

Puccinia sparganioides

Fraxinus pennsylvanica Marsh. var. lanceolata (Borkh.) Sarg.

Puccinia sparganioides

Fritillaria kamtschatcensis Ker.

Uromyces miurae

Galium aparine L.

Puccinia difformis

Galium asprellum Michx.

Puccinia punctata

Galium boreale L.

Puccinia rubefaciens

Galium trifidum L.

Puccinia punctata

Galium triflorum Michx.

Pucciniastrum galii

Puccinia punctata var. troglodytes

Gaylussacia baccata (Wang.) K. Koch

Pucciniastrum myrtilli

Gaura coccinea Nutt.

Uromyces plumbarius

Gentiana affinis Griesb.

Puccinia gentianae

Gentiana amarella L. var. acuta (Michx.) Herder

Puccinia haleniae

Gentiana andrewsii Gries.

Puccinia gentianae

Gentiana interrupta Greene

Puccinia gentianae

Gentiana strictiflora (Rydb.) A Nels.

Uromyces gentianae

Geranium maculatum L.

Puccinia polygoni-amphibii var. persicariae

Geranium spp.

Uromyces geranii

Gilia linearis (Nutt.) Gray

Uromyces acuminatus var. polemonii

Glaux maritima L.

Puccinia aristidae

P. distichlidis

Glyceria grandis Wats.

Puccinia graminis

P. rubigo-vera var. agropyrina

Glycyrrhiza lepidota Nutt.

Uromyces glycyrrhizae

Gnaphalium macounii Greene

Puccinia investita

Godetia spp.

Pucciniastrum pustulatum

Grindelia perennis Nels.

Puccinia grindeliae

Grindelia squarrosa (Pursh) Dunal

Puccinia grindeliae 
Grossularia cynosbati (L.) Mill.

Cronartium ribicola

Puccinia caricis var. grossulariata

$P$. caricis var. uniporula

Grossularia divaricata (Dougl.) Cov. \& Britt.

Cronartium ribicola

Melampsora ribesii-purpureae

Puccinia parkerae

Grossularia hirtella (Michx). Spach Puccinia caricis var. grossulariata

Grossularia setosa (Lindl.) Cov. \& Britt. Puccinia caricis var. grossulariata

Habenaria dilatata (Pursh) Hook. Puccinia praegracilis var. connersii

Habenaria gracilis Wats.

Aecidium graebnerianum

Puccinia praegracilis var. praegracilis

Habenaria psycodes (L.) Sw.

Aecidium graebnerianum

Hedysarum americanum (Michx.) Britt.

Uromyces hedysari-obscuri

Hedysarum boreale Nutt.

Uromyces hedysari-obscuri

Hedysarum cinerascens Rydb.

Uromyces hedysari-obscuri

Hedysarum mackenzii Richards.

Uromyces hedysari-obscuri

Hedysarum sulphurascens Rydb.

Uromyces hedysari-obscuri

Helianthus annuus L., cult.

Puccinia helianthi

Helianthus aridus Rydb.

Puccinia helianthi

Helianthus fascicularis Greene

Puccinia helianthi

Helianthus giganteus L.

Puccinia helianthi

Helianthus petiolaris Nutt.

Puccinia helianthi

Uromyces junci

Helianthus rigidus (Cass.) Desf.

Puccinia helianthi

Helianthus strumosus L.

Puccinia helianthi

Helianthus subrhomboideus Rydb.

Puccinia helianthi

Uromyces junci

Helianthus subtuberosus Bourg.

Puccinia helianthi

Uromyces junci

Helianthus tuberosus L.

Puccinia helianthi

Uromyces junci

Hepatica acutiloba DC.

Tranzschelia pruni-spinosae

Heuchera cylindrica Dougl. var. glabella (T. \& G.) Wheelock

Puccinia heucherae var. heucherae

Heuchera cylindrica var. trionalis R. B. \& L. Puccinia heucherae var. saxifragae
Heuchera glabra Willd.

Puccinia heucherae var. heucherae

Heuchera micrantha Dougl. ex Lindl.

Puccinia heucherae var. heucherae

Heuchera ovalifolia Nutt.

Puccinia heucherae var. heucherae

$P$. heucherae var. saxifragae

Heuchera richardsonii R. Br.

Puccinia heucherae var. saxifragae

Hieracium albiflorum Hook.

Aecidium columbiense

Hieracium canadense Michx. Puccinia hieracii

Hieracium scabriusculum Schw. Puccinia extensicola var. hieraciata P. hieracii

Hieracium scabrum Michx.

Puccinia fraseri

P. hieracii

Hierochloë odorata (L.) Wahl.

Puccinia coronata

Holcus lanatus L.

Puccinia coronata

$P$. rubigo-vera var. agropyri

Honkenya peploides (L.) Ehrh.

Puccinia arenariae

Hordeum caespitosum Scribn.

Puccinia glumarum

Hordeum jubatum L.

Puccinia glumarum

P. graminis

P. montanensis

P. rubigo-vera var. agropyri

P. rubigo-vera var. agropyrina

P. rubigo-vera var. impatientis

Hordeum murinum L., cult.

Puccinia graminis

Hordeum vulgare L., cult.

Puccinia graminis

P. hordei (P. anomala)

$\mathrm{P}$. glumarum

Houstonia longifolia Gaertn.

Uromyces houstoniatus

Hypericum kalmianum L.

Uromyces hyperici

Hypericum perforatum L. Uromyces hyperici

Hypericum prolificum L. Uromyces hyperici

Hypericum virginicum L. (Triadenum v. (L.) Raf.)

Uromyces hyperici

U. sparganii

Hystrix patula Moench

Puccinia rubigo-vera var. agropyrina

Impatiens balsamina $\mathrm{L}$.

Cronartium flaccidum

Impatiens biflora Walt.

Puccinia argentata

P. rubigo-vera var. impatientis

Impatiens noli-tangere $\mathrm{L}$.

Puccinia argentata 
Impatiens pallida Nutt.

Puccinia argentata

P. rubigo-vera var. impatientis

Iris versicolor $\mathrm{L}$.

Puccinia iridis

$P$. sessilis

Iris $\mathrm{sp}$.

Puccinia iridis

Iva axillaris Pursh

Puccinia intermixta

Juncus ater Rydb.

Uromyces junci

Juncus balticus Willd.

Uromyces junci

U. junci-effusi

Juncus longistylis Torr.

Uromyces silphii

Juncus tenuis Willd.

Uromyces silphii

Juncus xiphioides E. Mey.

Uromyces junci-effusi

Juniperus communis L.

Gymnosporangium clavipes

G. clavariaeforme

Juniperus fragrans (cult.)

Gymnosporangium globosum

Juniperus horizontalis Moench

Gymnosporangium corniculans

G. juvenescens

G. globosum

G. clavipes

Juniperus scopulorum Sarg.

Gymnosporangium nelsoni

$\mathrm{G}$. betheli

Juniperus sibirica Burgsd.

Gymnosporangium clavipes

G. juniperinum

Juniperus virginiana $L$.

Gymnosporangium clavipes

G. globosum

G. nidus-avis

G. juniperi-virginianae

G. corniculans

Juniperus spp.

Gymnosporangium clavipes

G. globosum

Koeleria cristata (L.) Pers.

Puccinia koeleriae

P. monoica

Lactuca pulchella (Pursh) DC.

Puccinia extensicola var. hieraciata

P. minussensis

Lactuca sativa L., cult.

Puccinia extensicola var. hieraciata

Lamarkia aurea (L.) Moench

(Achyrodes a Ktze.)

Puccinia coronata

Lappula echinata Gilib.

Puccinia aristidae
Lapsana communis L.

Puccinia lapsanae

Larix laricina (DuRoi) Koch

Melampsora bigelowii

M. medusae

Larix lyallii Parl.

Melampsora bigelowii

Lathyrus myrtifolius Muhl.

Uromyces fabae

Lathyrus ochroleucus Hook.

Uromyces fabae

Lathyrus venosus Muhl.

Uromyces fabae

Ledum glandulosum Nutt.

Chrysomyxa ledi var. glandulosi

Ledum groenlandicum Oeder

Chrysomyxa ledi var. groenlandici

C. ledicola

C. woronini

Ledum palustre L. var. decumbens Ait

Chrysomyxa ledi var. ledi

C. ledicola

C. woronini

Lespedeza capitata Michx.

Uromyces lespedezae-procumbentis

Lewisia rediviva Pursh

Uromyces spragueae

Liatris aspera (Michx.) Greene

Puccinia liatridis

Liatris ligulistylis (A. Nels.) Rydb.

Puccinia liatridis

Liatris punctata Hook.

Puccinia liatridis

Ligusticum apiifolium (Nutt.) Gray

Puccinia ligustici

Ligusticum scothicum L.

Aecidium ligustici

Lilium canadense L.

Uromyces holwayi

Lilium columbianum Hanson Uromyces holwayi

Lilium michiganense Farwell Uromyces holwayi

Lilium umbellatum Pursh Puccinia sporoboli

Limonium latifolium Uromyces limonii

Limonium nashii Small (not L. carolinianum (Walt.) Britt.)

Uromyces limonii-caroliniani

Limonium vulgare Uromyces limonii

Linum lewisii Pursh Melampsora lini

Linum rigidum Pursh Melampsora lini

Linum usitatissimum L., cult. Melampsora lini

Lobelia syphilitica L. Puccinia lobeliae

Lolium multiflorum Lam. Puccinia graminis 
Lolium perenne L.

Puccinia graminis

Lonicera caerulea L.

Puccinia festucae

Lupinus perennis $\mathrm{L}$.

Puccinia andropogonis var. onobrychidis

Lupinus sp.

Uromyces lupini

Luzula campestris (L.) DC. var. multiflora (Ehrh.) Celak.

Puccinia obscura

Luzula carolinae Wats.

Puccinia obscura

Luzula parviflora (Ehrh.) Desv.

Puccinia obscura

Lycopus americanus Muhl.

Puccinia angustata

Lycopus asper Greene

Puccinia angustata

Lycopus rubellus Moench

Puccinia angustata

Lycopus uniflorus Michx.

Puccinia angustata

Lycopus virginicus L.

Puccinia angustata

Lygodesmia juncea (Pursh) D. Don

Puccinia extensicola var. hieraciata

P. grindeliae

P. stipae

Lysimachia thyrsiflora L.

Puccinia limosae

Madia sativa Molina

Coleosporium madiae

Mahonia aquifolium (Pursh) Nutt.

Puccinia koeleriae

Mahonia repens (Lindl.) Don

Puccinia koeleriae

Mahonia sp.

Puccinia graminis

Cumminsiella sanguinea

Maianthemum canadense Desf.

Puccinia amphigena

P. sessilis

Uromyces acuminatus var. magnatus

Malus coronaria (L.) Mill.

Gymnosporangium juniperi-virginianae

Malus pumila Mill.

Gymnosporangium clavipes

G. globosum

G. juniperi-virginianae

Malus rivularis (Doug.) Roem.

Gymnosporangium nootkatense

Malus sylvestris (L.) Mill.

Gymnosporangium juniperi-virginianae

Malva moschata L.

Puccinia malvacearum

Malva rotundifolia $\mathrm{L}$.

Pucinia malvacearum

Malva spp.

Puccinia malvacearum

Medicago lupulina L.

Uromyces striatus
Medicago sativa $\mathrm{L}$.

Uromyces striatus

Melampyrum lineare Lam.

Puccinia adropogonis var. melampyri

Mentha arvensis L.

Puccinia angustata

P. menthae

Mentha canadensis $L$.

Puccinia angustata

P. menthae

Mentha glabrior (Hook.) Rydb.

Puccinia angustata

P. menthae

Mentha piperita L. Puccinia menthae

Mentha spicata L. Puccinia menthae

Merckia physodes Fisch. Puccinia arenariae

Micranthes lyallii (Engler) Small Puccinia heucherae var. heucherae

Milium effusum L. Puccinia graminis

Mitella breweri Gray Puccinia heucherae var. austroberingiana

Mitella diphylla L. Puccinia heucherae var. heucherae

Mitella nuda L.

Aecidium mitellae

Puccinia heucherae var. heucherae

Mitella ovalis Greene

Puccinia heucherae var. heucherae

Mitella trifida Graham

Puccinia heucherae var. austroberingiana

Moehringia lateriflora (L.) Fenzl

Puccinia arenariae

Uromyces acuminatus var. spartinae

Monarda didyma L.

Puccinia menthae

Monarda fistulosa L.

Puccinia menthae

Monarda menthaefolia Benth. Puccinia menthae

Monarda mollis L.

Puccinia menthae

Monarda mollis var. menthaefolia Fern Puccinia menthae

Moneses uniflora (L.) Gray

Chrysomyxa monesis

C. pirolata

Pucciniastrum pyrolae

Muhlenbergia cuspidata (Torr.) Rydb. Puccinia schedonnardi

Muricauda dracontium (L.) Small Uromyces caladii

Myrica gale L.

Cronartium comptoniae

Nabalus albus (L.) Hook.

Puccinia extensicola var. hieraciata

Nabalus altissimus (L.) Hook.

Puccinia orbicula 
Nabalus racemosus (Michx.) DC.

Puccinia maculosa

P. orbicula

Nabalus trifoliolatus Cass.

Puccinia orbicula

Nabalus sp.

Puccinia orbicula

Naumburgia thyrsiflora (L.) Duby

Puccinia limosae

Nemexia herbacea (L.) Small

Puccinia amphigena

Nemexia lasioneuron (Hook.) Rydb. Puccinia amphigena

Oenothera biennis L.

Puccinia extensicola var. oenotherae

Onoclea sensibilis L.

Uredinopsis mirabilis

Onopordum acanthium L.

Puccinia onopordi

Onosmodium occidentale Mackenzie

Puccinia rubigo-vera var. a pocrypta

Oryzopsis asperifolia Michx.

Puccinia pygmaea

Osmorrhiza claytoni (Michx.) Clarke

Puccinia pimpinellae

Osmorrhiza longistylis (Torr.) DC.

Puccinia pimpinellae

Osmunda claytoniana L.

Uredinopsis osmundae

Osmunda spectabilis Willd.

Uredinopsis osmundae

Oxyria digyna (L.) Hill.

Puccinia oxyriae

Oxytropis gracilis (A. Nels.) K. Schum.

Uromyces punctatus

Padus nana (DuRoi) Roem.

Tranzschelia pruni-spinosae

Panicum capillare $\mathrm{L}$.

Puccinia emaculata

Parnassia fimbriata Banks

Puccinia parnassiae

Parnassia palustris L.

Puccinia uliginosa

Pentstemon acuminatus Dougl.

Puccinia andropogonis var. pentstemonis

Pentstemon albidus Nutt.

Puccinia andropogonis var. pentstemonis

Pentstemon eriantherus Pursh

Puccinia andropogonis var. pentstemonis

Pentstemon nitidus Dougl.

Puccinia andropogonis var. pentstemonis

Petalostemon candidus (Willd.) Michx.

Puccinia andropogonis var. onobrychidis

Petalostemon oligophyllus (Torr.) Rydb.

Uropyxis petalostemonis

Petalostemon purpureus (Vent.) Rydb. Puccinia andropogonis var. onobrychidis

Petasites frigidus (L.) Fries

Puccinia conglomerata

Petasites palmatus (Ait.) A. Gray

Puccinia conglomerata
Phaca frigida L.

Uromyces lapponicus

Phacelia franklinii ( $\mathrm{R} . \mathrm{Br}$.) Gray

Puccinia rubigo-vera var. apocrypta

Phacelia heterophylla Pursh

Puccinia rubigo-vera var. apocrypta

Phalaris arundinacea $\mathrm{L}$.

Puccinia sessilis

Phalaris canariensis $\mathrm{L}$. Puccinia graminis

Phaseolus vulgaris $L$. Uromyces phaseoli

Phegopteris dryopteris (L.) Fée Uredinopsis phegopteridis Hyalopsora aspidiotus

Phleum pratense L.

Puccinia graminis var. phlei-pratensis

Phlox hoodii Richards.Puccinia douglasii

Phlox sp. Uromyces acuminatus var. polemonii

Phragmites communis Trin. Puccinia magnusiana P. phragmitis

Physalis heterophylla Nees Puccinia physalidis

Physalis virginiana Mill. Puccinia physalidis

Picea engelmanni (Parry) Engelm.

Chrysomyxa empetri

C. ledicola

C. weirii

Melampsorella cerastii

Picea excelsa (Lam.) Link

Melampsorella cerastii

Picea glauca (Moench) Voss (P. canadensis (Mill.) B.S.P.)

Chrysomyxa chiogenis

C. empetri

C. ledi var. cassandrae

C. ledicola

C. pirolata

Melampsorella cerastii

Pucciniastrum arcticum

P. americanum

Picea glauca var. albertiana (S. Brown) Sarg. (P. albertiana S. Brown.)

Chrysomyxa ledicola

C. pirolata

Melampsorella cerastii

Picea mariana (Mill.) B.S.P.

Chrysomyxa chiogenis

C. empetri

C. ledi

C. ledi var. groenlandici

C. ledicola

C. pirolata

C. woronini

Melampsorella cerastii

Picea pungens Engelm.

Chrysomyxa ledi

C. ledicola 
Picea rubens Sarg.

Chrysonyxa empetri

C. ledi

C. ledi var. cassandrae

C. ledi var. groenlandica

C. ledicola

C. weirii

Picea sitchensis (Bong.) Carr.

Chrysomyxa ledicola

C. monesis

C. weiri

Picea sp.

Chrysomyxa weirii

Pinus banksiana Lamb. Coleosporium solidaginis

Cronartium comandrae

C. comptoniae

Pinus contorta Dougl.

Coleosporium solidaginis

Cronartium coleosporioides

C. comandrae

Pinus ponderosa Dougl.

Cronartium coleosporioides

C. comandrae

Pinus spp.

Cronartium comandrae

C. ribicola

Pisum sativum L.

Uromyces fabae

Plantago eriopoda Torr.

Puccinia aristidae

Uromyces peckianus

Pleurogyne rotata Griesb.

Uromyces gentianae

Poa annua L.

Puccinia poae-nemoralis

Poa arida Vasey

Puccinia rubigo-vera var. agropyri

Poa compressa $\mathrm{L}$.

Puccinia graminis

$P$. poae-nemoralis

Poa crocata Michx.

Puccinia poae-nemoralis

Uromyces dactylidis

Poa interior Rydb.

Puccinia poae-nemoralis

Poa nemoralis $\mathrm{L}$.

Puccinia poae-nemoralis

Poa palustris L.

Puccinia poae-nemoralis

Poa pratensis L.

Puccinia poae-nemoralis

Uromyces dactylidis

Poa sandbergii Vasey

Puccinia rubigo-vera var. agropyri

Poa trivialis L.

Uromyces dactylidis

Poa spp.

Puccinia graminis var. poae

P. poae-nemoralis

Podophyllum peltatum L.

Puccinia podophylli
Polygala paucifolia Willd.

Puccinia pyrolae

Polygala senega L.

Puccinia andropogonis var. polygalina

Polygonum aviculare $\mathrm{L}$.

Uromyces polygoni

Polygonum buxiforme Small

Uromyces polygoni

Polygonum coccineum (Muhl.) Pers.

Puccinia polygoni-amphibii var. persicariae

Polygonum convolvulus L.

Puccínia polygoni-amphibii var. convolvuli

Polygonum erectum L.

Puccinia aristidae

Uromyces polygoni

Polygonum fowleri Rob.

Uromyces polygoni

Polygonum harwrightii Gray

Puccinia polygoni-amphibii var. persicariae

Polygonum hydropiperoides (Michx.) Small Puccinia polygoni-amphibii var. persicariae

Polygonum lapathifolium L.

Puccinia polygoni-amphibii var. persicariae

Polygonum muhlenbergii (Meisn.) Wats.

Puccinia polygoni-amphibii var. persicariae

Polygonum neglectum Besser

Puccinia aristidae

Polygonum pensylvanicum L.

Puccinia polygoni-amphibii var. persicariae

Polygonum persicaria L.

Puccinia polygoni-amphibii var. persicariae

Polygonum ramosissimum Michx.

Uromyces polygoni

Polygonum rubescens Small

Uromyces polygoni

Polygonum spergulariaeforme Meissn.

Uromyces polygoni

Polypodium virginianum $\mathrm{L}$.

Milesia polypodophila

Populus acuminata Rydb.

Melampsora occidentalis

Populus balsamifera L. (P. tacamahacca Mill.)

Melampsora medusae

M. occidentalis

Populus deltoides Marsh.

Melampsora medusae

Populus grandidentata Michx.

Melampsora abietis-canadensis

Populus sargentii Dode

Melampsora medusae

Populus termuloides Michx.

Melampsora abietis-canadensis

M. albertensis

M. medusae 
Populus trichocarpa T. \& G. Melampsora occidentalis

Populus spp.

Melampsora medusae

Potentilla bipinnatifida Dougl.

Phragmidium ivesiae

P. potentillae

Potentilla canadensis L.

Frommea obtusa

Potentilla concinna Richards.

Phragmidium ivesiae

Potentilla diversifolia Lehm.

Phragmidium ivesiae

Potentilla flabelliformis Lehm. Phragmidium ivesiae

Potentilla fruticosa L.

Phragmidium andersoni

Potentilla glabrella Rydb.

Phragmidium potentillae

Potentilla glaucophylla Lehm. Phragmidium ivesiae

Potentilla glomerata Nels. Phragmidium ivesiae

Potentilla hippiana Lehm. Phragmidium potentillae $P$. ivesiae

Potentilla nuttallii Lehm. Phragmidium ivesiae

Potentilla pectinata Raf. Phragmidium potentillae

Potentilla pennsylvanica L. Phragmidium potentillae

Potentilla strigosa Pall. Phragmidium potentillae

Potentilla tridentata Ait. (Sibbaldiopsis t.) Pucciniastrum potentillae

Potentilla spp.

Phragmidium invesiae

P. potentillae

Prenanthes, see Nabalus

Prunus domestica L.

Tranzschelia pruni-spinosae

Pseudotsuga taxifolia (Poir.) Britt. (P. mucronata (Raf.) Sudw.)

Melampsora albertensis

M. occidentalis

Psilactis asteroides Gray Puccinia asteris

Psoralea argophylla Pursh Uromyces psoraleae var. argophyllae

Psoralea lanceolata Pursh Uromyces psoraleae var. psoraleae

Psoralea physodes Dougl. Uromyces abbreviatus

Pteridium aquilinum pubescens Underw. Uredinopsis macrosperma

Pteretis nodulosa (Michx.) Nieuwl. Uredinopsis struthiopteridis

Puccinellia maritima (Huds.) Parl. Puccinia rubigo-vera var. agropyrina

Puccinellia nuttalliana (Schult.) Hitchc. Puccinia rubigo-vera var. agropyri
Puccinellia tenuiflora (Griesb.) Scribn. \& Merr. Puccinia rubigo-vera var. agropyri

P. rubigo-vera var. agropyrina

Pulsatilla ludoviciana (Nutt.) Heller Tranzschelia suffusca Puccinia pulsatillae

Pycnanthemum flexuosum (Walt.) B.S.P. Puccinia menthae

Pycnanthemum virginianum (L.) Dur. \& Jacks. Puccinia menthae

Pyrola americana Sweet Chrysomyxa pirolata

Pyrola asarifolia Michx.

Chrysomyxa pirolata

Pucciniastrum pyrolae

Pyrola bracteata Hook.

Chrysomyxa pirolata

Pyrola chlorantha Sw. (P. virens Schweigg.)

Chrysomyxa pirolata

Pucciniastrum pyrolae

Pyrola elliptica Nutt.

Chrysomyxa pirolata

Pucciniastrum pyrolae

Pyrola grandifolia Radius

Chrysomyxa pirolata

Pyrola minor L.

Chrysomyxa pirolata

Pyrola picta Smith

Chrysomyxa pirolata

Pyrola rotundifolia Michx.

Pucciniastrum pyrolae

Pyrola secunda L.

Chrysomyxa pirolata

Pucciniastrum pyrolae

Pyrola uliginosa T. \& G.

Chrysomyxa pirolata

Pyrus communis L.

Gymnosporangium clavariaeforme

Pyrus sp.

Gymnosporangium clavipes

Ranunculus abortivus L.

Puccinia eatoniae var. ranunculi

Ranunculus acris L.

Puccinia rubigo-vera var. agropyrina

Ranunculus affinis R.Br.

Puccinia rubigo-vera var. agropyrina

Ranunculus cariophyllus Hook.

Puccinia rubigo-vera var. agropyrina

Ranunculus cymbalaria Pursh

Puccinia rubigo-vera var. agropyri

$P$. rubigo-vera var. agropyrina

Ranunculus macounii Britt.

Uromyces alopecuri

Ranunculus micranthus Nutt.

Uromyces alopecuri

Ranunculus recurvatus Poir.

Tranzschelia pruni-spinosae

Ranunculus repens L.

Uromyces dactylidis

Ranunculus sceleratus L.

Uromyces alopecuri 
Ranunculus suksdorfii Gray

Puccinia ranunculi

Rhamnus alnifolia L'Her.

Puccinia coronata var. calamagrostis

Rhamnus cathartica L.

Puccinia coronata var. avenae

Rhamnus davurica Pall.

Puccinia coronata

Rhamnus frangula L.

Puccinia coronata var. agrostis

Rhamnus purshiana DC.

Puccinia coronata

Rhamnus utilis Decne.

Puccinia coronata

Rheum rhaponticum L.

Puccinia phragmites

Rhododendron lapponicum (L.) Wahl. Chrysomyxa ledi var. rhododendri

Rhodora canadensis (L.) Carr. Pucciniastrum myrtilli

Rhus aromatica Ait. Pileolaria toxicodendri

Rhus radicans $\mathrm{L}$.

Pileolaria toxicodendri

Rhus toxicodendron auct. Pileolaria toxicodendri

Rhynchospora alba (L.) Vahl Uromyces rhyncosporae

Ribes americanum Mill.

Puccinia caricis var. grossulariata

Ribes bracteosum Dougl.

Cronartium ribicola

Puccinia caricis var. grossulariata

Ribes echinatum Lindl.

Cronartium ribicola

Melampsora ribesii-purpureae

Puccinia parkerae

Ribes glandulosum Gauer

Puccinia caricis var. grossulariata

Ribes grossularia L.

Cronartium ribicola

Puccinia caricis var. grossulariata

Ribes hudsonianum Richards.

Puccinia caricis var. grossulariata

Ribes lacustre (Pers.) Poir.

Puccinia caricis var. grossulariata

P. parkerae

Ribes laxiflorum Pursh

Puccinia caricis var. grossulariata

Ribes nigrum L.

Cronartium ribicola

Puccinia caricis var. grossulariata

Ribes setosum Lindley

Puccinia caricis var. grossulariata

Ribes triste Pallas

Puccinia ribis

Ribes spp.

Cronartium ribicola

Puccinia caricis var. grossulariata

Rosa acicularis Lindl.

Phragmidium rosae-acicularis
Rosa blanda Ait.

Phragmidium americanum

P. montivagum

P. speciosum

Rosa bourgeauiana Crépin

Phragmidium montivagum

$P$. rosae-acicularis

Rosa californica C. \& S.

Phragmidium rosae-californicae

Rosa gymnocarpa Nutt.

Phragmidium rosae-californicae

Rosa macounii Greene

Phragmidium speciosum

Rosa nutkana Presl

Phragmidium rosae-acicularis

P. rosae-californicae

Rosa pisocarpa Gray

Phragmidium rosae-californicae

Rosa suffulta Greene

Phragnidium rosicola

P. rosae-acicularis

P. rosae-arkansanae

Rosa virginiana Mill.

Phragmidium americanum

P. speciosum

Rosa spp.

Phragmidium americanum

P. disciflorum

P. rosae-pimpinellifoliae

P. rosae-arkansanae

$P$. rosae-acicularis

P. rosae-californicae

P. rosicola

P. speciosum

P. subcorticinum

P. montivagum

Rubus acaulis Michx.

Gymnoconia peckiana

Pucciniastrum arcticum

Rubus arcticus L.

Gymnoconia peckiana

Pucciniastrum arcticum

Rubus idaeus L. var. strigosus Maxim. Phragmidium rubi-idaei

Rubus leucodermis Dougl. Phragmidium rubi-idaei

Rubus melanolasius Focke Pucciniastrum americanum Phragmidium rubi-idaei

Rubus nigrobaccus Bailey Gymnoconia peckiana

Rubus occidentalis L. Phragmidium rubi-idaei

Rubus odoratus L.

Phragmidium rubi-odorati

Rubus parviflorus Nutt. Phragmidium occidentale

Rubus procumbens Muhl. Kuenhneola uredinis

Rubus pubescens Raf.

Kuehneola uredinis

Pucciniastrum arcticum 
Rubus randii (Bailey) Rydb. Gymnoconia peckiana

Rubus strigosus Michx. Phragmidium rubi-idaei Pucciniastrum americanum

Rubus triflorus Richards. Gymnoconia peckiana Puccinastrum arcticum

Rubus spp.

Gymnoconia peckiana Phragmidium rubi-idaei Pucciniastrum americanum $P$. arcticum

Rudbeckia ampla A. Nels. Uromyces perigynius

Rudbeckia laciniata L. Uromyces perigynius U. rudbeckiae

Rumex britannica L. Puccinia ornata

Rumex mexicanus Meisn. Puccinia phragmitis

Rumex occidentalis S. Wats. Puccinia ornata P. phragmitis

Rumex sp. Puccinia ornata

Salicornia herbacea L. Uromyces peckianus

Salicornia pacifica Standl. Uromyces peckianus

Salix alba $L$. var. vitellina $L$.

Melampsora abieti-capraearum

Salix amygdaloides Anders. Melampsora bigelowii

Salix anglorum Cham. Melampsora arctica

Salix arbusculoides Anders. Melanpsora arctica

Salix balsamifera (Hook.) Barratt Melampsora arctica

Salix bebbiana Sarg.

Melampsora abieti-capraearum M. ribesii-purpurea

Salix candida Flugge Melampsora abieti-capraearum

Salix cordata Muhl.

Melampsora abieti-capraearum

Salix glaucops Anders. Melampsora arctica

Salix myrtillifolia Anders. Melampsora arctica

Salix petrophila Rydb. Melampsora arctica

Salix purpurea L. var. gracilis Anders. Melampsora abieti-capraearım

Salix rubra Richards. Melampsora abieti-capraearum

Salix spp. Melampsora bigelowii M. arctica
Sanicula marilandica L. Puccinia marylandica

Sanicula sp.

Puccinia marylandica

Sarcobatus vermiculatus (Hook.) Torr. Puccinia aristidae

Saxifraga aizoides $\mathrm{L}$. Puccinia pazschkei var. jueliana

Saxifraga bronchialis L.

Puccinia pazschkei var. tricuspidatae

Saxifraga cernua L.

Puccinia heucherae var. saxifragae

Saxifraga ferruginea Graham

Puccinia pazschkei var. jueliana

Saxifraga hieracifolia Waldst. \& Kit.

Puccinia heucherae var. saxifragae

Saxifraga lyallii Engler (Micranthes 1. (Engler) Small

Puccinia heucherae var. heucherae

Saxifraga mertensiana Bong.

Puccinia pazschkei var. heterisiae

Saxifraga nivalis $\mathrm{L}$.

Puccinia heucherae var. saxifragae

Saxifraga occidentalis S. Wats. ssp. rufidulus (Small) Basigalupi

Puccinia heucherae var. saxifragae

Saxifraga oppositifolia L.

Puccinia fischeri

P. pazschkei var. oppositifoliae

Saxifraga punctata sspp.

Puccinia heucherae var. austroberingiana

Saxifraga rivularis $\mathrm{L}$.

Puccinia heucherae var. saxifragae

Saxifraga tenuis (Wallr.) Sm.

Puccinia heucherae var. saxifragae

Saxifraga tricuspidata Rottb. (Leptasea $t$. (Rottb.) Haw.)

Puccinia pazschkei var. tricuspidatae

Saxifraga virginiensis Michx.

Puccinia heucherae var. saxifragae

Scirpus atrovirens Muhl.

Puccinia angustata

Scirpus cyperinus (L.) Kunth

Puccinia angustata

Scirpus fluviatilis (Torr.) Gray

Uromyces scirpi

Scirpus microcarpus Presl

Puccinia angustata

P. mcclatchieana

Scirpus paludosus A.Nels.

Uromyces scirpi

Scirpus validus Vahl

Puccinia obtecta

Uromyces scirpi

Scolochloa festucacea (Willd.) Link

Puccinia coronata

Sedum stenopetalum Pursh

Puccinia rydbergii

Secale cereale $\mathrm{L}$.

Puccinia graminis

P. rubigo-vera var. secalis

Sempervivum tectorum L.

Endophyllum sempervivi 
Senecio columbianus Greene

Puccinia extensicola var. hieraciata

Senecio flarulus Greene

Puccinia extensicola var. hieraciata

Senecio triangularis Hook.

Puccinia angustata

P. subcircinata

Senecio willingii Greenm.

Puccinia recedens

Shepherdia argentea Nutt.

Puccinia caricis-shepherdiae

Shepherdia canadensis (L.) Nutt.

Puccinia caricis-shepherdiae

P. coronata

Sibbaldiopsis tridentata (Sol.) Rydb.

Pucciniastrum potentillae

Sideranthus spinulosus (Pursh) Sweet

Puccinia grindeliae

Sisymbrium altissimum L.

Puccinia aristidae

Sium cicutaefolium Schrank

Uromyces scirpi

Smilacina stellata (L.) Desf.

Puccinia amphigena

$P$. sessilis

Uromyces acuminatus var. magnatus

Smilax herbacea L.

Puccinia amphigena

Solidago canadensis L.

Coleosporium solidaginis

Solidago gilvocanescens (Rydb.) Smyth Coleosporium solidaginis

Solidago glaberrima Mart. Puccinia stipae

Solidago glabrum Mart. Coleosporium solidaginis

Solidago mollis Bartl.

Puccinia stipae

Solidago multiradiata Ait. Coleosporium solidaginis

Solidago nemoralis Ait.

Puccinia extensicola var. solidaginis

Solidago patula Muhl.

Coleosporium solidaginis

Solidago puberula Nutt. Puccinia virgaureae

Solidago rigida $\mathrm{L}$.

Puccinia extensicola var. solidaginis

P. stipae

Solidago serotina Ait.

Coleosporium solidaginis

Puccinia extensicola var. solidaginis

Solidago uliginosa Nutt.

Coleosporium solidaginis

Solidago sp.

Puccinia stipae

Sorbus americana Marsh.

Gymnosporangium aurantiacum (G. cornutum)

Sorbus occidentalis (Wats.) Greene

Gymnosporangium nootkatense

G. juniperinum (G. tremelloides)
Sorbus scopulina Greene

Gymnosporangium aurantiacum (G. cornutum)

G. juniperinum (G. tremelloides)

Sorbus sitchensis Roem.

Gymnosporangium juniperinum (G. tremelloides)

Sparganium eurycarpum Engelm.

Uromyces sparganii

Spartina alterniflora Loisel.

Uromyces acuminatus

Spartina gracilis Trin.

Puccinia distichlidis

Uromyces acuminatus

Spartina pectinata Link (S. michauxiana Hitchc.)

Puccinia distichlidis

P. seymouriana

P. sparganioides

Uromyces acuminatus

Spartina spp.

Puccinia distichlidis

Spergularia canadensis (Pers.) D. Don

Uromyces acuminatus var. spartinae

Sphaeralcea coccinea (Nutt.) Rydb.

Puccinia sherardiana

Sphenopholis obtusata (Michx.) Scribn.

Puccinia eatoniae

Sphenopholis pallens (Spreng.) Scribn.

Puccinia eatoniae

Spinacia oleracea L.

Puccinia aristidae

Sporobolus cryptandrus (Torr.) Gray

Puccinia graminis

Steironema ciliatum (L.) Raf.

Puccinia dayi

P. distichlidis

Stellaria graminea L.

Melampsorella cerastii

Stellaria longifolia Muhl.

Puccinia arenariae

Stellaria longipes Goldie

Puccinia arenariae

Melampsorella cerastii

Stipa comata Trin. \& Rupr.

Puccinia stipae

Stipa spartea Trin.

Puccinia stipae

Stipa tweedyi Scribn. \& Vasey

Puccinia stipae

Stipa viridula Trin.

Puccinia scaber

Suaeda maritima (L.) Dumort

Uromyces peckianus

Symphoricarpos albus (L.) Blake

Puccinia crandallii

P. symphoricarpi

Symphoricarpos occidentalis Hook.

Puccinia crandallii

Symphoricarpos spp.

Puccinia crandallii 
Taraxacum dumetorum Greene

$$
\text { Puccinia hieracii }
$$

Taraxacum kok-saghyz Rod. Puccinia hieracii

Taraxacum vulgare (Lam.) Schr. Puccinia hieracii $P$. variabilis

Tellima grandiflora (Pursh) Dougl. Puccinia heucherae var. austroberingiana

Thalictrum alpinum $L$.

Puccinia rubigo-vera var. agropyri

Thalictrum dasycarpum Fisch. \& Lall. Puccinia rubigo-vera var. agropyrina Tranzschelia thalictri

Thalictrum dioicum $\mathrm{L}$.

Puccinia rubigo-vera var. agropyri

Thalictrum polygamum Muhl.

Tranzschelia thalictri

Thalictrum venulosum Trel.

Puccinia rubigo-vera var. agropyri

$P$. rubigo-vera var. agropyrina

Tranzschelia thalictri

Thalictrum sp.

Tranzschelia thalictri

Thlaspi arvense $\mathrm{L}$.

Puccinia aristidae

Tiarella cordifolia L.

Puccinia heucherae var. heucherae

Tiarella unifoliata Hook.

Puccinia heucherae var. heucherae

Tolmiea menziesii (Pursh) T. \& G.

Puccinia heucherae var. heucherae

Trautvetteria grandis Nutt.

Puccinia pulsatillae

Trientalis americana Pursh

Puccinia karelica

Trifolium dubium Sibth.

Uromyces minor

Trifolium hybridum L.

Uromyces nerviphilus

U. trifolii var. hybridi

Trifolium incarnatum L.

Uromyces trifolii var. trifolii-repentis

Trifolium medium L.

Uromyces trifolii var, fallens

Trifolium microdon H. \& A.

Uromyces minor

Trifolium oliganthum Steud. Uromyces minor

Trifolium pratense I.

Uromyces trifolii var. fallens

U. nerviphilus

Trifolium repens $L$.

Uromyces nerviphilus

U. trifolii var. trifolii-repentis

Triglochin maritima L.

Puccinia aristidae

Triodia flava (L.) Smyth

Puccinia windsoriae

Trisetum spicatum (L.) Ricker

Puccinia monoica
Triticum aestivum $\mathrm{L}$.

Puccinia glumarum

P. graminis

P. rubigo-vera var. tritici

Triticum dicoccum Schrank.

Puccinia graminis

Triticum durum Desf.

Puccinia graminis

P. rubigo-vera var. tritici

Triticum spelta $L$.

Puccinia rubigo-vera var. tritici

Tsuga canadensis (L.) Carr.

Melampsora abietis-canadensis

M. farlowii

Pucciniastrum myrtilli

Tsuga heterophylla (Raf.) Sarg.

Uraecium holwayi

Tsuga mertensiana (Bong.) Carr.

Uraecium holwayi

Tussilago farfara L.

Puccinia poarum

Urtica dioica L.

Puccinia caricis var. urticata

Urtica gracilis Ait.

Puccinia caricis var. urticata

Urtica lyallii Wats.

Puccinia caricis var. urticata

Vaccinium corymbosum L.

Pucciniastrum myrtilli

Vaccinium myrtillus $\mathrm{L}$.

Pucciniastrum myrtilli

$P$. goeppertianum

Vaccinium oreophilum Rydb.

Puccinisatrum goeppertianum

Vaccinium parvifolium Smith

Chrysomyxa ledi var. vaccinii

Vaccinium pennsylvanicum Lam.

Pucciniastrum myrtilli

P. goeppertianum

Vaccinium vitis-idaea $L$.

Pucciniastrum goeppertianum

Vaccinium spp.

Pucciniastrum goeppertianum

Valeriana septentrionalis Rydb.

Puccinia commutata

Valeriana sitchensis Bong.

Puccinia commutata

Veratrum californicum Durand

Puccinia veratri

Veratrum eschscholtzianum (R. \& S.) Rydb.

Puccinia veratri

Veronica alpina L. var. unalaschcensis C. \& S.

(V. wormskjoldii R. \& S.)

Puccinia albulensis

Viburnum cassinoides L.

Coleosporium viburni

Viburnum lentago L.

Coleosporium viburni

Viburnum pauciforum Pylaie

Puccinia linkii 
Vicia americana Muhl.

Uromyces coloradensis var. campester

U. coloradensis var. maritimus

U. fabae

Vicia angustifolia Reichard

Uromyces fabae

Vicia cracca L.

Uromyces coloradensis var. campester

U. fabae

Vicia oregana Nutt.

Uromyces coloradensis var. campester

Vicia sativa $L$.

Uromyces fabae

Vicia sparsifolia Nutt.

Uromyces coloradensis var. campester

U. fabae

Vinca major L.

Puccinia vincae

Viola adunca Smith

Puccinia violae

Viola canadensis L.

Puccinia violae

P. ornatula

Viola conspersa Reichenb.

Puccinia viola

Viola eriocarpa Schw.

Puccinia violae

Viola glabella Nutt.

Puccinia ornatula

Viola langsdorfii Fisch.

Puccinia fergussoni

Viola mackloskeyi Lloyd

Puccinia fergussoni

Viola nephrophylla Greene

Puccinia ellisiana

P. fergussoni

P. violae

Viola odorata L.

Puccinia violae
Viola orbiculata Geyer

Puccinia canadensis

Viola pedatifida G. Don.

Puccinia violae

Viola pubescens Ait.

Puccinia violae

Viola renifolia Gray Puccinia violae

Violae rugulosa Greene Puccinia ornatula

Viola septentrionalis Greene Puccinia violae

Viola sororia Willd. Puccinia violae

Violae tricolor $\mathrm{L}$.

Puccinia violae

Viola spp.

Puccinia ellisiana

P. violae

Waldsteinia fragarioides (Michx.) Tratt. Puccinia waldsteiniae

Xanthium commune Britt. Puccinia xanthii

Xanthium chinense Mill. Puccinia xanthii

Zea mays L.

Puccinia sorghi

Zizia aurea (L.) Koch

Puccinia angelicae

Zizia cordata (Walt.) Koch

Puccinia ziziae

Zygadenus chloranthus Richards. Puccinia atropuncta

Zygadenus elegans Pursh Puccinia grumosa

Zygadenus gramineus Rydb. Uromyces zygadeni 


\section{REFERENCES}

1. Arthur, J. C. Manual of the rusts in United States and Canada. Lafayette, Purdue Research Foundation, 1934. 438 p.

2. Bisby, G. R., and others. The fungi of Manitoba and Saskatchewan. Ottawa, National Research Council, 1938. p. 62-73.

3. Canadian Plant Disease Survey, 11th report, 1931. Ottawa, Canada, Dept. of Agriculture, Experimental Farms Branch.

4. Same, 12 th report, 1932.

5. Same, 13th report, 1933.

6. Same, 14 th report, 1934.

7. Same, 16th report, 1936.

8. Same, 17th report, 1937.

9. Same, 18th report, 1938.

13. Same, 19th report, 1939.

11. Same, 20th report, 194 n.

12. Same, 21st report, 1941.

13. Same, 22nd report, 1942.

14. Same. 23rd report, 1943.

15. Same, 24th report, 1944.

16. Same, 25th report, 1945.

17. Same, 26th report, 1946.

18. Same, 27th report, 1947.

19. Same, 28th report, 1948.

20. Same, 29th report, 1949.

21. Same, 30th report, 1950.

22. Same, 31st report, 1951.

23. Check list of diseases of economic plants in Canada. Comp. by Ivan H. Crowell and E. Lavallée with the cooperation of the Common Names Committee, Canadian Phytopathological Society and the Check List Committee, Quebec Society for the Protection of Plants, 1942. Ottawa, Canada, Dept. of Agriculture, Science Service. n.d. 68 p.

24. Drayton, F. L., Comp. A summary of the prevalence of plant diseases in the Dominion of Canada, 1920-1924. Ottawa, Canada, Dept. of Agriculture, Dominion Experimental Farms, Div. of Botany. 1926. 88 p. Bull. 71, n.s.

25. Fraser, W. P. and I. L. Conners. The Uredinales of the Prairie Provinces of Western Canada. Roy. Soc. Can. Trans. 3d series, v. 19, sect. 5. p. 279-308. 1925.

26. Moss, E. H. Rusts on Adoxa in Alberta. Mycologia 43:99-102. 1951.

27. Savile, D. B. O. North American species of Chrysomyxa. Can. J. Research, C, 28:318-330. 1950.

28. Savile, D. B. O. A new rust on Deschampsia. Mycologia 42:663-667. 1950.

29. Savile, D. B. O. The relationship of Puccinia praegracilis and P. connersii. Mycologia 43:456-458. 1951.

30. Savile, D. B. O. and I. L. Conners. The rusts of Armeria and Limonium in North America. Mycologia 43:186-195. 1951.

31. Wehmeyer, L. E. Contributions to a study of the fungous flora of Nova Scotia, I. p.242-243. Papers Mich. Acad. Sci. Arts and Letters. 20:233-266. 1934.

\section{Addendum}

32. Faull, J. H. Two spruce-infecting rusts: Chrysomyxa Piperiana and Chrysomyxa Chiogenis. J. Arnold Arboretum 17:109-114. 1936.

33. Faull, J. H. Chrysomyxa Empetri-A spruce-infecting rust. J. Arnold Arboretum 18:141-148. 1937.

34. Savile, D. B. O. Taxonomy, phylogeny, host relationship and phytogeography of the microcyclic rusts of Saxifragaceae. Can. J. Botany 32:400-425. 1954.

35. Savile, D. B. O. Chrysomyxa in North America-additions and corrections. Can. J. Botany 33:487-496. 1955.

36. Ziller, W. G. Studies of western tree rusts. I. A new cone rust on Sitka spruce. Can. J. Botany 32:432-439. 1954 .

37. Ziller, W: G. Studies of western tree rusts. II. Melampsora occidentalis and M. albertensis, two needle rusts of Douglas fir. Can. J. Botany 33:177-188. 1955. 



EDMOND CLOUTIER, C.M.G., O.A., D.S.P.

QUEEN'S PRINTER AND CONTROLLER OF STATIONERY OTTAWA, 1850 